\title{
The effect of a non-zero Lagrangian time scale on bounded shear dispersion
}

\author{
Matthew S. Spydell $\dagger$ and Falk Feddersen
}

Integrative Oceanography Division, Scripps Institution of Oceanography, La Jolla, CA 92093-0209, USA

(Received 20 January 2011; revised 28 September 2011; accepted 6 October 2011; first published online 13 December 2011)

Previous studies of shear dispersion in bounded velocity fields have assumed random velocities with zero Lagrangian time scale (i.e. velocities are $\delta$-function correlated in time). However, many turbulent (geophysical and engineering) flows with mean velocity shear exist where the Lagrangian time scale is non-zero. Here, the longitudinal (along-flow) shear-induced diffusivity in a two-dimensional bounded velocity field is derived for random velocities with non-zero Lagrangian time scale $\tau_{L}$. A non-zero $\tau_{L}$ results in two-time transverse (across-flow) displacements that are correlated even for large (relative to the diffusive time scale $\tau_{D}$ ) times. The longitudinal (along-flow) shear-induced diffusivity $D_{S}$ is derived, accurate for all $\tau_{L}$, using a Lagrangian method where the velocity field is periodically extended to infinity so that unbounded transverse particle spreading statistics can be used to determine $D_{S}$. The non-dimensionalized $D_{S}$ depends on time and two parameters: the ratio of Lagrangian to diffusive time scales $\tau_{L} / \tau_{D}$ and the release location. Using a parabolic velocity profile, these dependencies are explored numerically and through asymptotic analysis. The large-time $D_{S}$ is enhanced relative to the classic Taylor diffusivity, and this enhancement increases with $\sqrt{\tau_{L}}$. At moderate $\tau_{L} / \tau_{D}=0.1$ this enhancement is approximately a factor of 3 . For classic shear dispersion with $\tau_{L}=0$, the diffusive time scale $\tau_{D}$ determines the time dependence and large-time limit of the shear-induced diffusivity. In contrast, for sufficiently large $\tau_{L}$, a shear time scale $\tau_{S}=\left(\tau_{L} \tau_{D}\right)^{1 / 2}$, anticipated by a simple analysis of the particle's domain-crossing time, determines both the $D_{S}$ time dependence and the large-time limit. In addition, the scalings for turbulent shear dispersion are recovered from the large-time $D_{S}$ using properties of wall-bounded turbulence.

Key words: mixing, mixing and dispersion

\section{Introduction}

A sheared mean current can greatly enhance the dispersion of a passive tracer ('scalar') in the direction perpendicular to the shear. This phenomenon is called shear dispersion and was first investigated for laminar Poiseuille flow in pipes (Taylor 1953). For laminar flow in a two-dimensional (2D) pipe, tracer evolves following the

$†$ Email address for correspondence: mspydell@ucsd.edu 
advection-diffusion equation

$$
\frac{\partial c}{\partial t}+U \frac{\partial c}{\partial x}=K \nabla^{2} c
$$

where $c$ is the tracer concentration, $t$ is time, $x$ is the longitudinal (alongflow) direction, $U$ is the longitudinal velocity, $K$ is the diffusivity, and $\nabla^{2}$ is the two-dimensional Laplacian operator. Instead of explicitly solving (1.1), Taylor heuristically derived a simpler diffusion equation for the transverse (across-flow)averaged concentration $C(x, t)$

$$
\frac{\partial C}{\partial t}+U_{0} \frac{\partial C}{\partial x}=D_{e f f}^{\infty} \frac{\partial^{2} C}{\partial x^{2}},
$$

where

$$
C(x, t)=\frac{1}{L} \int_{0}^{L} c(x, y, t) \mathrm{d} y \quad \text { and } \quad U_{0}=\frac{1}{L} \int_{0}^{L} U(y) \mathrm{d} y,
$$

with $y$ the transverse direction and $L$ the width of the transverse domain. The effective diffusivity

$$
D_{\text {eff }}^{\infty}=K+D_{T}^{\infty}
$$

has two contributions: the small-scale longitudinal (in the flow direction) diffusivity $K$ and the (shear-induced) Taylor diffusivity $D_{T}^{\infty}$. For a parabolic current with (transverseaveraged) mean velocity $U_{0}, U=6 U_{0} y(L-y) / L^{2}$, the Taylor diffusivity is

$$
D_{T}^{\infty}=U_{0}^{2} \tau_{D} / 210
$$

where the diffusive time scale is $\tau_{D}=L^{2} / K$. It should be noted that the diffusion equation for transverse-averaged concentration (1.2) is an asymptotic result valid only for times $t \gg \tau_{D}$ when tracer concentration is Gaussian and the dispersion is independent of the initial concentration distribution. By reducing the number of independent variables needed to describe the longitudinal dispersion, Taylor's theory is both elegant and practical. As such, this theory has been used by scientists and engineers from a variety of disciplines (including hydrology, physiology, chemical engineering, meteorology, oceanography, etc.) to study, model and understand tracer dispersion (Young \& Jones 1991).

Taylor's derivation of (1.2) assumed that the Péclet number $P e=U_{0} L / K$ is large so that $D_{T}^{\infty} \gg K$. However, the large Péclet number assumption is unnecessary and the tracer spreading rate, i.e. the effective (shear-induced) diffusivity can be found using the so called method of moments (e.g. Aris 1956). Using this method, Barton (1983) properly derived expressions, valid for all time, for the effective spreading rate $D_{T}\left(t, y_{0}\right)$ that depend upon time and initial tracer release location $y_{0}$. In the large-time limit, the Taylor asymptotic diffusivity was recovered, i.e. $D_{T}^{\infty}=\lim _{t \rightarrow \infty} D_{T}\left(t, y_{0}\right)$.

Generalized dispersion is often studied from a Lagrangian perspective. Taylor (1922) showed that the particle spreading rate (diffusivity) depends only on Lagrangian velocity autocovariance function. For times small compared with the Lagrangian velocity decorrelation time, i.e. the Lagrangian time scale $\tau_{L}$, the dispersion is ballistic and the mean-squared displacement in unbounded and homogeneous flows is quadratic in time $\left\langle[\Delta x(t)]^{2}\right\rangle=\overline{u^{2}} t^{2}$, where $\overline{u^{2}}$ is the mean-squared random velocity. For $t \gg \tau_{L}$ the dispersion is Brownian and $\left\langle[\Delta x(t)]^{2}\right\rangle=2 \kappa t$ where the constant diffusivity is given by $\kappa=\overline{u^{2}} \tau_{L}$. For molecular diffusion the Lagrangian time scale is so small, $\tau_{L} \approx O\left(10^{-7} \mathrm{~s}\right) \quad$ (Li et al. 2010), it is generally assumed to be zero. However, this 
assumption is unphysical and leads to undefined velocities. Hence, a complete theory of molecular particle dispersion requires a finite $\tau_{L}$ (Uhlenbeck \& Ornstein 1930). Turbulent dispersion is also characterized by ballistic and Brownian dispersion regimes despite a different mechanism of dispersion, namely finite-sized eddies. In turbulent dispersion the Lagrangian time scale $\tau_{L}$ and eddy diffusivity $\kappa$ are much larger than their molecular counterparts. Thus, a more complete view of bounded turbulent shear dispersion, requires a non-zero $\tau_{L}$.

The majority of shear dispersion investigations are Eulerian and based upon the Fickian diffusion equation (e.g. Taylor 1953; Aris 1956, and others). However, shear dispersion is also investigated from a Lagrangian perspective. In particular, using the probability distribution function (p.d.f.) of particle displacements in a bounded domain, Van Den Broeck (1982), and more recently Camassa, Lin \& McLaughlin (2010), reproduced the Eulerian shear-dispersion results (e.g. Barton 1983). These results were also obtained by Haber \& Mauri (1988) using a similar method, but with displacement p.d.f.s in an infinite transverse domain rather than a bounded domain. These Lagrangian analyses of shear dispersion (e.g. Van Den Broeck 1982; Dewey \& Sullivan 1982; Haber \& Mauri 1988; Camassa et al. 2010) assumed that the random particle velocities were $\delta$-correlated in time, i.e. $\tau_{L}=0$ in these studies.

For laminar flows in the smallest microchannels $\left(L \approx 10^{-6} \mathrm{~m}\right)$, using values of $K \approx 10^{-10} \mathrm{~m}^{2} \mathrm{~s}^{-1}$ and $\tau_{L} \approx 10^{-12} \mathrm{~s}$ for the diffusion of fluorescein (a tracer commonly used in laboratory studies) in water, the ratio $\tau_{L} / \tau_{D}$ is very small, about $10^{-10}$ and Lagrangian time scale effects can be neglected. However, numerous mean-sheared turbulent geophysical flows have $\tau_{L} / \tau_{D} \approx O\left(10^{-1}\right)$. Although the following geophysical flows are inhomogeneous, using representative published values for the transverse eddy-diffusivity $(\kappa)$, the transverse scale of the mean current $(L)$, and transverse Lagrangian time scale $\left(\tau_{L}\right)$, the ratio $\tau_{L} / \tau_{D}$ can be estimated. In the surfzone, $\tau_{L} / \tau_{D}$ is as large as 0.5 (Spydell, Feddersen \& Guza 2009), for surface currents in the Santa Barbara Channel it is as large as 0.1 (Dever, Hendershott \& Winant 1998), as large as 0.5 for the Gulf Stream extension (McClean, Poulain \& Pelton 2002), as large as 0.3 for the Kuroshio extension (Zhurbas 2003), as large as 0.25 for the California Current (Davis 1985), and as large as 0.1 for the Antarctic Circumpolar Current (Sallée et al. 2008). For these flows, the effective longitudinal diffusivity may be affected by the presence of correlated random transverse motions (i.e. $\tau_{L} \neq 0$ ).

The effect of non-zero $\tau_{L}$ on shear dispersion in various regimes has been examined. For unbounded shear dispersion with $U(y)=\alpha y$, the effect of $\tau_{L}$ is transient and disappears for $t \gg \tau_{L}$ (Zambianchi \& Griffa 1994; Katayama \& Terauti 1996) with the large-time shear-induced diffusivity given by $D_{\text {eff }}^{\infty} \propto K^{2} t^{2}$. This effective diffusivity is fundamentally different than the bounded $\tau_{L}=0$ shear flow version $D_{T}^{\infty}=U_{0}^{2} L^{2} / 210 K$. For a steady square integrable velocity field, i.e. a quasibounded velocity field, the effective shear-induced diffusivity increases with the Lagrangian time scale (Castiglione \& Crisanti 1999), although a complete expression for the effective diffusivity's dependence upon time and $\tau_{L}$ was not considered. Numerical simulations of particle dispersion in steady and time-dependent Taylor-Green flow show that the effective diffusivity increases with increasing $\tau_{L}$ (Pavliotis \& Stuart 2005; Pavliotis, Stuart \& Zygalakis 2007). An expression for the effective long-time diffusivity for a broad class of flows (for zero and non-zero $\tau_{L}$ ) was determined using the method of homogenization. For the flows considered, the expression for the $\tau_{L} \neq 0$ case is not easily solved and an explicit formula for the effective diffusivity's dependence on $\tau_{L}$ is not given. However, for sinusoidal shear flow $(U(y)=\sin (y))$ and small $\tau_{L}$, Pavliotis, Stuart \& Zygalakis (2009) showed asymptotically that the 
long-time effective diffusivity increases with $\sqrt{\tau_{L}}$ relative to $\tau_{L}=0$. Moreover, this scaling was confirmed by Monte Carlo simulations of particle trajectories.

The effect of a non-zero Lagrangian time scale on shear dispersion may be important for bounded flows, or square integrable flows, when $\tau_{L}$ is not very small compared with the diffusive time scale $\tau_{D}$. Thus, the problem of shear dispersion in a bounded two-dimensional sheared velocity field with a non-zero Lagrangian time scale is considered here. In particular, the expression for the particle spreading rate, the effective diffusivity, as a function of release location $y_{0}$, time since release $t$ and Lagrangian time scale $\tau_{L}$ is derived. The derivation follows that of Haber \& Mauri (1988), but allows for non-zero $\tau_{L}$. The coordinate system, the Langevin equations for particle position and velocity, and the averaging methods are defined in $\S 2$. Unbounded particle statistics for non-zero $\tau_{L}$ in the transverse (across-flow) direction (parallel to the velocity shear) are considered in $\S 3$. For non-zero $\tau_{L}$, the correlation of successive transverse particle displacements becomes non-zero and must be accounted for in deriving the longitudinal (along-flow) shear-induced diffusivity. The transverse particle statistics are used to derive the $\tau_{L} \neq 0$ shear-induced longitudinal particle dispersion statistics, including the Lagrangian autocovariance function and the shearinduced effective diffusivity $D_{S}(t)(\S 4)$.

The non-zero $\tau_{L}$ derivation for the shear-induced diffusivity is for any squareintegrable velocity profile. Properties of the shear-induced diffusivity are examined using a parabolic velocity profile $(\$ 5)$, in order to compare the results with Taylor's theory $\left(\tau_{L}=0\right)$. The shear-induced diffusivity normalized by the Taylor diffusivity, $D_{S}(t) / D_{T}^{\infty}$, is a function of time $t$ and depends on two non-dimensional parameters: the ratio $\tau_{L} / \tau_{D}$ and the normalized release location. The dependence of $D_{S}(t)$ on each of these parameters is examined separately. Of particular note, the ratio $D_{S}^{\infty} / D_{T}^{\infty}$ (where $D_{S}^{\infty}$ is the $D_{S}(t)$ large-time limit) is enhanced $(\geqslant 1)$ and increases with $\tau_{L} / \tau_{D}$. At $\tau_{L} / \tau_{D}=0.1$ the ratio $D_{S}^{\infty} / D_{T}^{\infty} \approx 3$, a significant deviation from Taylor's result. For moderately sized $\tau_{L}$, asymptotic analysis of the shear-induced diffusivity reveals the leading-order time scale and large-time limit of $D_{S}(t)(\S 6)$. In contrast to a time scale of $\tau_{D}$ and large-time diffusivity $D_{T}^{\infty} \propto U_{0}^{2} \tau_{D}$ for $\tau_{L}=0$, the $\tau_{L} \neq 0$ time scale is given by $\tau_{S}=\left(\tau_{D} \tau_{L}\right)^{1 / 2}$ and the large-time diffusivity is $D_{S}^{\infty} \sim U_{0}^{2} \tau_{S}$. The time scale transition from $\tau_{D}$ to $\tau_{S}$ can be anticipated by considering the particle's transverse-crossing time (§7.1). In addition, $D_{S}^{\infty}$ is shown to be consistent with expressions ( $D^{\infty} \propto u_{*} L$, where $u_{*}$ is a friction velocity) found for wall-bounded turbulent shear dispersion $(\S 7.2)$. The main results are summarized in $\S 8$.

\section{Langevin equations}

Shear dispersion in a two-dimensional $(x, y)$ domain is studied using Langevin equations for particle velocities and displacements. The longitudinal (along-flow) and transverse (across-flow) directions are $x$ and $y$, respectively. The imposed velocity $U$ is only in the $x$ direction and depends only on $y$, thus, $U=U(y)$ (see figure 1). This velocity is analogous to the mean velocity for turbulent flows. The domain is bounded by walls at $y=0$ and $L$, thus distances will be scaled by the domain width $L$ throughout.

Particle evolution is governed by the Langevin equations

$$
\frac{\mathrm{d} u}{\mathrm{~d} t}=-\frac{u}{\tau_{L}}+\sqrt{\frac{2 \overline{u^{2}}}{\tau_{L}}} w_{x}
$$




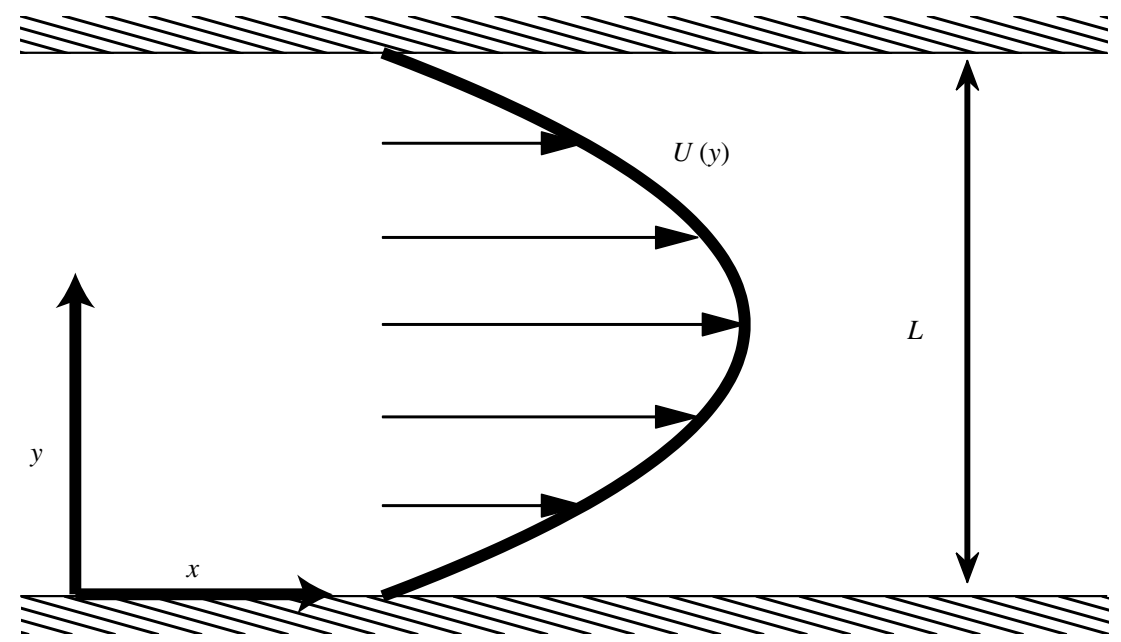

FIGURE 1. A schematic diagram of the flow domain with walls at $y=0$ and $L$. The imposed velocity $U(y)$ is in the longitudinal direction, $x$, and only depends on transverse coordinate $y$.

$$
\begin{gathered}
\frac{\mathrm{d} v}{\mathrm{~d} t}=-\frac{v}{\tau_{L}}+\sqrt{\frac{2 \overline{v^{2}}}{\tau_{L}}} w_{y}+\eta_{B} \\
\frac{\mathrm{d} x}{\mathrm{~d} t}=U(y)+u \\
\frac{\mathrm{d} y}{\mathrm{~d} t}=v
\end{gathered}
$$

where $(x, y)$ in these equations refers to the particle position. The random particle velocities are $u$ and $v$ in the $x$ and $y$ directions, respectively, and have variance denoted by $\overline{u^{2}}$ and $\overline{v^{2}}$ and it is assumed that $\overline{u^{2}}=\overline{v^{2}}$ for simplicity. The $W_{i}$ are independent incremental Wiener processes such that $W_{i}(t)=\int_{0}^{t} w_{i}\left(t^{\prime}\right) \mathrm{d} t^{\prime}$ so $\mathrm{d} W_{i}(t)=w(t) \mathrm{d} t$ where $w(t)$ is a zero-mean, stationary, white-noise process with variance (squared ensembleaveraged) $\left\langle w_{i}(t) w_{j}\left(t^{\prime}\right)\right\rangle=\delta\left(t-t^{\prime}\right) \delta_{i j}$. To account for particle reflection at the boundaries $y=(0, L)$, an additional 'noise' term $\eta_{B}$ is included in $(2.1 b)$ resulting in no particle flux through the boundaries (Van Den Broeck 1982).

Particle velocities are correlated in time with the memory of earlier velocities over the Lagrangian time scale $\tau_{L}$. More realistic Lagrangian models for inhomogeneous turbulence used to investigate atmospheric boundary layer dispersion, and basin-scale oceanic dispersion, can include spatially varying Lagrangian time scales and velocity variances (e.g. Wilson \& Sawford 1996; Berloff \& McWilliams 2002). Similar to Lagrangian models of dispersion in turbulent uniform shear flows (e.g. Sawford \& Yeung 2001), here $\tau_{L}, \overline{u^{2}}$ and $\overline{v^{2}}$ are constant in the transverse direction, to isolate and analytically examine the effect of $\tau_{L}$ on shear dispersion. The Lagrangian time scale $\tau_{L}$ is set equal in both directions. Unique transverse and longitudinal Lagrangian time scales only slightly alter the analysis that follows. Note that for $\tau_{L}=0$, the set of four Langevin equations (2.1) is replaced by two equations for the $(x, y)$ position with noise added directly to the positions (e.g. Zambianchi \& Griffa 1994) such that positions perform a classical random walk. In this case, the p.d.f. of particle position is governed by (1.1). 
Before addressing the statistics of (2.1), different averages need to be defined and their notation introduced. Consider the velocity time series of a particle $v(t)$. The ensemble-averaged velocity of particles that have an initial velocity $v_{0}=v(t=0)$ is denoted by

$$
\left\langle v\left(t \mid v_{0}\right)\right\rangle \text { : ensemble averaging } v \text { with same initial velocity } v_{0}
$$

and is considered a subensemble (or conditional) average as all trajectories have the same initial velocity. Ensemble averaging the velocity over all trajectories with any initial velocity is given by

$$
\langle v(t)\rangle \text { : indicates ensemble averaging } v \text { over all possible initial velocities. }
$$

This average is considered the ensemble average in contrast to the subensemble average for which each trajectory has the same initial velocity. Averaging a particular quantity, for example the initial velocity $v_{0}$, is indicated by an overline

$$
\overline{v_{0}} \text { : indicates averaging the quantity } v_{0} \text { over all possible values }
$$

and was used in $(2.1 a, b)$ to indicate the random velocity variance.

\section{Unbounded transverse particle statistics}

In this section, the transverse (across-flow) particle dispersion statistics for a non-zero Lagrangian time scale are developed. Although bounded in the transverse direction, the domain can be assumed unbounded for the purposes of determining the effective longitudinal diffusivity (Haber \& Mauri 1988) since the transverse displacement statistics of $(2.1 b, d)$ in an infinite $y$ domain can be used to calculate the shear-induced longitudinal statistics (i.e. shear dispersion). Although the transverse statistics in the unbounded domain are different from those in the bounded domain, the analysis of transverse motions with $\tau_{L} \neq 0$ is greatly simplified as $\eta_{B}=0$ in $(2.1 b)$ and the difficulty of integrating $(2.1 b)$ in the bounded domain (e.g. Camassa et al. 2010) is avoided.

Many of the transverse statistics that follow were investigated and derived in Uhlenbeck \& Ornstein (1930) and are stated here without derivation. However, one derivation appears in appendix A to introduce the reader to the techniques from which all of the statistics that follow can be derived. The subensemble-averaged velocity of a particle is found by integrating $(2.1 b)$ with $\eta_{B}=0$ (see appendix A) and averaging

$$
\left\langle v\left(t \mid v_{0}\right)\right\rangle=v_{0} \exp \left(-t / \tau_{L}\right),
$$

where $v_{0}=v(t=0)$ is the initial velocity. As the average over all initial velocities is zero, $\overline{v_{0}}=0$, the ensemble-averaged velocity is

$$
\langle v(t)\rangle=\overline{v_{0}} \exp \left(-t / \tau_{L}\right)=0 .
$$

The subensemble-averaged squared velocity $\left\langle v\left(t_{1} \mid v_{0}\right) v\left(t_{2} \mid v_{0}\right)\right\rangle$ is found by integrating (2.1b) once to $t_{1}$, to obtain $v\left(t_{1} \mid v_{0}\right)$, and then again to $t_{2}$ to obtain $v\left(t_{2} \mid v_{0}\right)$ and then ensemble averaging the product (see appendix A for the complete derivation) resulting in

$$
\left\langle v\left(t_{1} \mid v_{0}\right) v\left(t_{2} \mid v_{0}\right)\right\rangle=\left(v_{0}^{2}-\overline{v^{2}}\right) \mathrm{e}^{-\left(t_{1}+t_{2}\right) / \tau_{L}}+\overline{v^{2}} \mathrm{e}^{-\left|t_{2}-t_{1}\right| / \tau_{L}} .
$$

When averaged over all possible initial velocities, such that $\overline{v_{0}^{2}}=\overline{v^{2}}$, the Lagrangian velocity autocovariance function is obtained

$$
\left\langle v\left(t_{2}\right) v\left(t_{1}\right)\right\rangle=\overline{v^{2}} \mathrm{e}^{-\left|t_{2}-t_{1}\right| / \tau_{L}} .
$$


Since the noise $w(t)$ is stationary, this function only depends on the time difference $t_{2}-t_{1}$. Many statistics will depend on the two times $t_{1}$ and $t_{2}$, and henceforth $t_{2}>t_{1}$ is assumed, although (3.4) is good for all $t_{1}$ and $t_{2}$.

The subensemble-averaged particle position is found by integrating (2.1b) twice and averaging (see appendix A)

$$
\left\langle y\left(t_{1} \mid v_{0}, y_{0}\right)\right\rangle=y_{0}+v_{0} \tau_{L}\left(1-\mathrm{e}^{-t_{1} / \tau_{L}}\right),
$$

where $y_{0}=y(t=0)$ is the initial transverse particle location. Thus, the subensembleaveraged position is the integral of the subensemble-averaged velocity (3.1). The first displacement is defined as $\Delta y_{1} \equiv y\left(t_{1}\right)-y(0)$ and its subensemble average is

$$
\left\langle\Delta y_{1}\left(t_{1} \mid v_{0}\right)\right\rangle=\left\langle y\left(t_{1}\right)-y(0)\right\rangle=v_{0} \tau_{L}\left(1-\mathrm{e}^{-t_{1} / \tau_{L}}\right) .
$$

Using the same techniques (appendix A) for deriving (3.4), the subensemble-averaged squared first displacement is

$$
\left\langle\left[\Delta y_{1}\left(t_{1} \mid v_{0}\right)\right]^{2}\right\rangle=\kappa \tau_{L}\left(2 t_{1} / \tau_{L}+4 \mathrm{e}^{-t_{1} / \tau_{L}}-\mathrm{e}^{-2 t_{1} / \tau_{L}}-3\right)+v_{0}^{2} \tau_{L}^{2}\left(1-\mathrm{e}^{-t_{1} / \tau_{L}}\right)^{2},
$$

where the definition of the diffusivity $\kappa=\bar{v}^{2} \tau_{L}$ is used. The last term in (3.7) is the mean displacement's (3.6) contribution to $\left\langle\left[\Delta y_{1}\right]^{2}\right\rangle$.

The second displacement, needed to determine the effective longitudinal diffusivity, is defined as $\Delta y_{2} \equiv y\left(t_{2}\right)-y\left(t_{1}\right)$ (recall $\left.t_{2}>t_{1}\right)$ and its subensemble average is

$$
\left\langle\Delta y_{2}\left(t_{2}, t_{1} \mid v_{0}\right)\right\rangle=\left\langle y\left(t_{2}\right)-y\left(t_{1}\right)\right\rangle=v_{0} \tau_{L}\left(\mathrm{e}^{-t_{1} / \tau_{L}}-\mathrm{e}^{-t_{2} / \tau_{L}}\right) .
$$

The subensemble-averaged squared second displacement is

$$
\begin{aligned}
\left\langle\left[\Delta y_{2}\left(t_{2}, t_{1} \mid v_{0}\right)\right]^{2}\right\rangle= & \kappa \tau_{L}\left(2\left(t_{2}-t_{1}\right) / \tau_{L}+2 \mathrm{e}^{-\left(t_{1}+t_{2}\right) / \tau_{L}}\right. \\
& \left.+2 \mathrm{e}^{-\left(t_{2}-t_{1}\right) / \tau_{L}}-\mathrm{e}^{-2 t_{1} / \tau_{L}}-\mathrm{e}^{-2 t_{2} / \tau_{L}}-2\right) \\
& +v_{0}^{2} \tau_{L}^{2}\left(\mathrm{e}^{-t_{1} / \tau_{L}}-\mathrm{e}^{-t_{2} / \tau_{L}}\right)^{2}
\end{aligned}
$$

with the last term in (3.9) due to the mean second displacement.

Owing to the memory of previous velocities, i.e. a non-zero $\tau_{L}$, first and second displacements are correlated in a manner similar to velocity. The displacement analogue to the velocity autocovariance (3.3) is

$$
\begin{aligned}
\left\langle\Delta y_{2}\left(t_{2}, t_{1} \mid v_{0}\right) \Delta y_{1}\left(t_{1} \mid v_{0}\right)\right\rangle= & \kappa \tau_{L}\left(2 \mathrm{e}^{-t_{2} / \tau_{L}}+\mathrm{e}^{-2 t_{1} / \tau_{L}}-2 \mathrm{e}^{-t_{1} / \tau_{L}}\right. \\
& \left.-\mathrm{e}^{-\left(t_{1}+t_{2}\right) / \tau_{L}}-\mathrm{e}^{-\left(t_{2}-t_{1}\right) / \tau_{L}}+1\right) \\
& +v_{0}^{2} \tau_{L}^{2}\left(1-\mathrm{e}^{-t_{1} / \tau_{L}}\right)\left(\mathrm{e}^{-t_{1} / \tau_{L}}-\mathrm{e}^{-t_{2} / \tau_{L}}\right) .
\end{aligned}
$$

This statistic, not previously considered in the literature, is critical to determining the effective longitudinal diffusivity. For $\tau_{L}=0,\left\langle\Delta y_{1} \Delta y_{2}\right\rangle=0$ indicating that nonzero $\Delta y_{1}$ and $\Delta y_{2}$ covariance is entirely due to the non-zero Lagrangian time scale. Although the proof is omitted here, first and second displacements can be shown to be Gaussian from higher displacement moments (e.g. Uhlenbeck \& Ornstein 1930). Furthermore, as $\Delta y_{1}\left(t_{1} \mid v_{0}\right)+\Delta y_{2}\left(t_{2}, t_{1} \mid v_{0}\right)=\Delta y_{1}\left(t_{2} \mid v_{0}\right)$, the sum $\Delta y_{1}+\Delta y_{2}$ is also Gaussian. Thus, the displacements $\Delta y_{1}$, and $\Delta y_{2}$ are jointly Gaussian and correlated.

Although it is possible to calculate effective longitudinal statistics based upon subensemble-averaged transverse statistics, which would make them dependent on the initial velocity, the effective longitudinal statistics will be calculated from ensembleaveraged transverse statistics by averaging the subensemble-averaged statistics over all possible initial velocities. Thus, averaging (3.6) over $v_{0}$, the ensemble-averaged first 


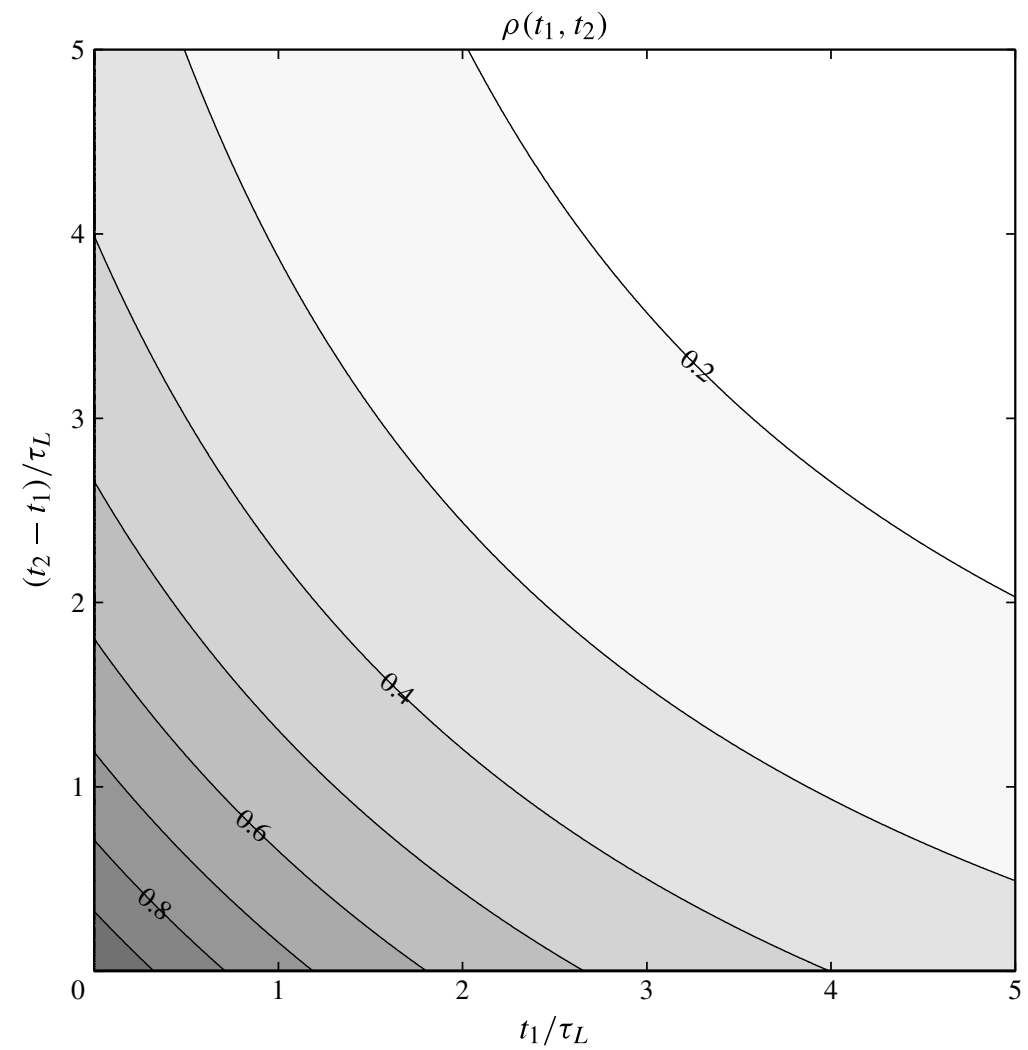

FIGURE 2. The correlation coefficient $\rho$ of first $\left(\Delta y_{1}\right)$ and second $\left(\Delta y_{2}\right)$ displacements as a function of $t_{1} / \tau_{L}$ and $\left(t_{2}-t_{1}\right) / \tau_{L}(3.12)$.

displacement is $\left\langle\Delta y_{1}\left(t_{1}\right)\right\rangle=0$ and similarly for the second displacement. However, the ensemble-averaged squared displacements are non-zero and are found by replacing $v_{0}^{2} \tau_{L}$ with $\kappa$ in (3.7), (3.9) and (3.10) since $\overline{v_{0}^{2}} \tau_{L}=\overline{v^{2}} \tau_{L}=\kappa$, hence

$$
\begin{gathered}
\sigma_{1}^{2}\left(t_{1}\right) \equiv\left\langle\left[\Delta y_{1}\left(t_{1}\right)\right]^{2}\right\rangle=2 \kappa\left(t_{1}+\tau_{L} \mathrm{e}^{-t_{1} / \tau_{L}}-\tau_{L}\right) \\
\sigma_{2}^{2}\left(t_{1}, t_{2}\right) \equiv\left\langle\left[\Delta y_{2}\left(t_{2}, t_{1}\right)\right]^{2}\right\rangle=2 \kappa\left(t_{2}-t_{1}+\tau_{L} \mathrm{e}^{-\left(t_{2}-t_{1}\right) / \tau_{L}}-\tau_{L}\right) \\
\sigma_{12}^{2}\left(t_{1}, t_{2}\right) \equiv\left\langle\Delta y_{1}\left(t_{1}\right) \Delta y_{2}\left(t_{2}, t_{1}\right)\right\rangle=\kappa \tau_{L}\left(1+\mathrm{e}^{-t_{2} / \tau_{L}}-\mathrm{e}^{-t_{1} / \tau_{L}}-\mathrm{e}^{-\left(t_{2}-t_{1}\right) / \tau_{L}}\right) .
\end{gathered}
$$

Note that the variances for $t_{1}>t_{2}$ are found by switching $t_{1}$ and $t_{2}$ in $(3.1 a-c)$.

The correlation coefficient between first and second displacements is given by

$$
\rho\left(t_{1}, t_{2}\right)=\frac{\sigma_{12}^{2}}{\sigma_{1} \sigma_{2}} .
$$

Thus, as with Lagrangian velocities, displacements are also correlated when $\tau_{L} \neq 0$ (figure 2). In particular, $\rho \approx 0.6$ when both displacement times are equal to the Lagrangian time scale $\left(t_{1} / \tau_{L}=\left(t_{2}-t_{1}\right) / \tau_{L}=1\right)$. Even at the relatively large displacement times of $t_{1} / \tau_{L}=\left(t_{2}-t_{1}\right) / \tau_{L}=3, \rho \approx 0.25$ as $\rho$ does not fall off exponentially like the Lagrangian velocity autocovariance function (3.4). The correlation coefficient $\rho$ has a symmetry about $t_{2}-t_{1}=t_{1}$ (figure 2), which can be shown explicitly by defining $\zeta=t_{1}$ and $\eta=t_{2}-t_{1}$, and noting that $\rho(\zeta, \eta)=\rho(\eta, \zeta)$. 

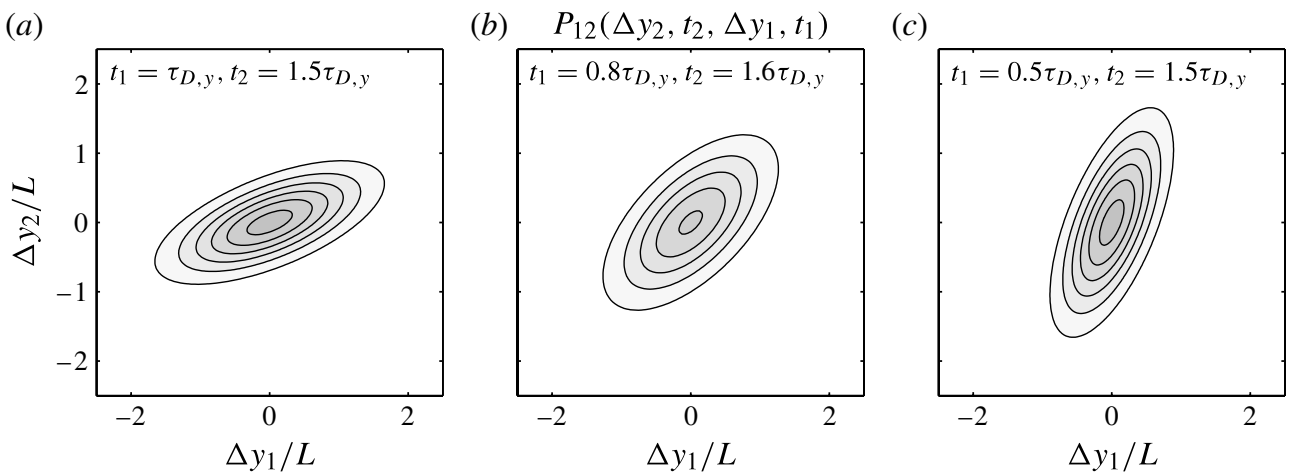

FIGURE 3. The unbounded $y$ domain joint p.d.f. $P_{12}$ as a function of $\Delta y_{1} / L$ and $\Delta y_{2} / L$ for three different combinations of $t_{1}$ and $t_{2}$ as indicated in each panel. In each panel, times were chosen such that $t_{1}^{2}+\left(t_{2}-t_{1}\right)^{2}=5 / 4$. The Lagrangian time scale is equal to the diffusive time $\tau_{L}=\tau_{D}$. Note that the contour intervals in each panel are equal and at an interval of 0.08 .

Previous investigations (e.g. Haber \& Mauri 1988) assumed uncorrelated velocities $\left(\tau_{L}=0\right)$, hence successive displacements were independent $(\rho=0)$.

The transverse particle spreading rate is the transverse effective diffusivity $D_{y}(t)$, defined by

$$
D_{y}(t) \equiv \frac{1}{2} \frac{\mathrm{d}}{\mathrm{d} t} \sigma_{1}^{2}(t)=\kappa\left(1-\mathrm{e}^{-t / \tau} L\right),
$$

and equal to the integral of the velocity autocovariance function $D_{y}(t)=$ $\int_{0}^{t}\left\langle v\left(t_{1}\right) v(t)\right\rangle \mathrm{d} t_{1}$, (e.g. Taylor 1922). For $t \rightarrow \infty$, the effective $y$-direction diffusivity is constant (Brownian motion) and given by $D_{y}=\kappa$. The diffusive time scale $\tau_{D}$, the time it takes particles to Brownian-diffuse across the transverse domain, is given by

$$
\tau_{D}=\frac{L^{2}}{\kappa}
$$

and is the natural time scale for bounded shear dispersion (e.g. Aris 1956).

Probability distribution functions of transverse displacements are required to determine effective longitudinal statistics. Since higher-order displacement moments indicate Gaussian displacements (Uhlenbeck \& Ornstein 1930), the p.d.f. of ensembleaveraged first displacements is

$$
P_{1}\left(\Delta y_{1}, t_{1}\right)=\frac{1}{\left(2 \pi \sigma_{1}^{2}\right)^{1 / 2}} \exp \left[-\frac{1}{2 \sigma_{1}^{2}} \Delta y_{1}^{2}\right] .
$$

Similarly, since first and second displacements are jointly Gaussian, the joint probability distribution function (j.p.d.f.) of ensemble-averaged first and second displacements is

$$
P_{12}\left(\Delta y_{2}, t_{2}, \Delta y_{1}, t_{1}\right)=\frac{1}{2 \pi \Sigma^{2}} \exp \left[-\frac{1}{2 \Sigma^{4}}\left(\sigma_{2}^{2} \Delta y_{1}^{2}+\sigma_{1}^{2} \Delta y_{2}^{2}-2 \sigma_{12}^{2} \Delta y_{1} \Delta y_{2}\right)\right] \text {, }
$$

where $\Sigma^{4}=\sigma_{1}^{2} \sigma_{2}^{2}-\sigma_{12}^{4}$. Because first and second displacements are not independent, the $\sigma_{12}^{2}$ term in the exponential of (3.16) rotates the contours of $P_{12}$, so that the major and minor axis of the ellipse, setting the terms in the exponential to a constant, are not parallel to the $\Delta y_{1}{ }^{-}$and $\Delta y_{2}$-axes. For $t_{2}-t_{1}$ less than $t_{1}$, the 
major axis of $P_{12}$ is rotated positively from the $\Delta y_{1}$-axis (figure $3 a$ ). When $t_{2}=2 t_{1}$ the major axis is rotated by exactly $\pi / 4$ (figure $3 b$ ) and when $t_{2}-t_{1}$ is larger than $t_{1}$ the rotation is greater than $\pi / 4$ (figure $3 c$ ) and approaches $\pi / 2$ as $t_{2} \gg t_{1}$. In contrast, for $\tau_{L}=0$ displacements are independent and $\sigma_{12}=0$, hence $P_{12}$ is the product of two independent Gaussians and therefore not rotated (e.g. Haber $\&$ Mauri 1988). Moreover, the bounded domain displacement j.p.d.f. for $\tau_{L}=0$ is also readily calculated as the product of independent (but not Gaussian) displacement p.d.f.s (Van Den Broeck 1982). In contrast, the $\tau_{L} \neq 0$ bounded domain j.p.d.f. is unknown, motivating the use of infinite domain statistics.

\section{Longitudinal particle statistics}

The same Lagrangian method of Haber \& Mauri (1988), used to calculate the Taylor diffusivity $D_{T}^{\infty}$, is used to calculate the non-zero Lagrangian time scale longitudinal statistics. Three primary longitudinal statistics are calculated: the mean Lagrangian velocity, the velocity autocovariance and the effective diffusivity.

\subsection{The mean Lagrangian longitudinal velocity}

From the Langevin equation (2.1c), the longitudinal particle velocity is $\dot{x}=\mathrm{d} x / \mathrm{d} t=$ $U(y)+u$. Thus, the ensemble-averaged longitudinal velocity of particles, the 'mean Lagrangian longitudinal velocity', with the original position of particles given by $y(t=0)=y_{0}$, is found from

$$
\left\langle\dot{x}\left(t \mid y_{0}\right)\right\rangle=\left\langle U\left(y\left(t \mid y_{0}\right)\right)+u(t)\right\rangle
$$

and has been previously called the 'Lagrangian mean-velocity history' (Dewey \& Sullivan 1982). Since the ensemble-averaged random velocity is zero, $\langle u(t)\rangle=0$, the mean Lagrangian longitudinal velocity is the expected value of the imposed velocity $U(y)$ that particles released from $y_{0}$ feel at time $t$, i.e.

$$
\left\langle\dot{x}\left(t \mid y_{0}\right)\right\rangle=\left\langle U\left(y\left(t \mid y_{0}\right)\right)\right\rangle .
$$

This is related to the p.d.f. of particle position

$$
\left\langle U\left(y\left(t \mid y_{0}\right)\right)\right\rangle=\int_{0}^{L} U(y) p_{1}\left(y, t \mid y_{0}\right) \mathrm{d} y
$$

where $p_{1}\left(y, t \mid y_{0}\right)$ is probability of finding particles at $y$ after time $t$ for particles that were originally at $y_{0}$ in the bounded $(0, L) y$ domain. The bounded domain p.d.f. is given by

$$
p_{1}\left(y, t_{1} \mid y_{0}\right)=\frac{1}{L}+\frac{2}{L} \sum_{n=1}^{\infty} \cos \left(\gamma_{n} y\right) \cos \left(\gamma_{n} y_{0}\right) \mathrm{e}^{-\frac{1}{2} \gamma_{n}^{2} \sigma_{1}^{2}},
$$

where $\gamma_{n}=n \pi / L$ and $\sigma_{1}^{2}$ is defined in (3.11a).

However, rather than working in the bounded domain, it is possible, and advantageous for calculating the effective longitudinal diffusivity, to work in a periodic transverse domain extended to infinity (also denoted as the infinite domain) by using the cosine series of $U(y)$

$$
U(y)=\sum_{n=0}^{\infty} U_{n} \cos \left(\gamma_{n} y\right),
$$


where $U_{0}=1 / L \int_{0}^{L} U(y) \mathrm{d} y$ is the transverse average of $U(y)$ and the Fourier coefficients for $n \geqslant 1$ are given by

$$
U_{n}=\frac{2}{L} \int_{0}^{L} U(y) \cos \left(\gamma_{n} y\right) \mathrm{d} y \text { for } n \geqslant 1 .
$$

In an infinite domain, (4.3) becomes

$$
\left\langle U\left(y\left(t \mid y_{0}\right)\right)\right\rangle=\int_{-\infty}^{\infty} \sum_{n=0}^{\infty} U_{n} \cos \left(\gamma_{n} y\right) \mathscr{P}_{1}\left(y, t \mid y_{0}\right) \mathrm{d} y,
$$

where $\mathscr{P}_{1}\left(y, t \mid y_{0}\right)$ is the probability of finding a particle at $y$ after time $t$ that was released at $y_{0}$. For the longitudinal statistics, periodically extending the velocity field to infinity with particles released in an unbounded domain is equivalent to having reflecting boundaries at $y=(0, L)$ since $U(-\delta)=U(\delta)$ and $U(L+\delta)=U(L-\delta)$ when $U(y)$ is given by (4.5) (Haber \& Mauri 1988). Hence, (4.7) is equivalent to (4.3).

Because transverse $(y)$ particle dispersion in an infinite domain is homogeneous, i.e. independent of position, $\mathscr{P}_{1}\left(y, t \mid y_{0}\right)=P_{1}\left(y-y_{0}, t\right)$, where $P_{1}$ is given by (3.15) since $\Delta y_{1}=y-y_{0}$. Thus, (4.7) becomes

$$
\left\langle U\left(y\left(t \mid y_{0}\right)\right)\right\rangle=\frac{1}{\sqrt{2 \pi \sigma_{1}^{2}(t)}} \int_{-\infty}^{\infty} \sum_{n=0}^{\infty} U_{n} \cos \left(\gamma_{n} y\right) \exp \left[-\frac{\left(y-y_{0}\right)^{2}}{2 \sigma_{1}^{2}(t)}\right] \mathrm{d} y,
$$

and performing the integral, the non-zero Lagrangian time scale mean longitudinal velocity is

$$
\left\langle\dot{x}\left(t \mid y_{0}\right)\right\rangle=\left\langle U\left(y\left(t \mid y_{0}\right)\right)\right\rangle=U_{0}+\sum_{n=1}^{\infty} U_{n} \cos \left(\gamma_{n} y_{0}\right) \exp \left[-\frac{\gamma_{n}^{2} \sigma_{1}^{2}(t)}{2}\right] .
$$

Note that using the bounded domain displacement p.d.f. (4.4) in (4.3) also gives (4.9). The expression (4.9) is valid even for $\tau_{L}=0$ and describes how the ensemble-averaged velocity of a particle transitions from $U\left(y_{0}\right)$, the velocity particles have at $t=0$, to $U_{0}$ the transverse mean imposed velocity. The large-time velocity is $U_{0}$ since all memory of the initial position of the particle is lost and a particle is equally likely to be at any transverse location. Note that (4.9) is only modestly different from the previously published expressions for $\tau_{L}=0$ (equation (15) in Haber \& Mauri 1988).

\subsection{The longitudinal velocity autocovariance}

The longitudinal velocity autocovariance is found in a similar manner to the mean Lagrangian longitudinal velocity. Again using $\dot{x}=\mathrm{d} x / \mathrm{d} t=U(y)+u$, the autocovariance is

$$
\left\langle\dot{x}\left(t_{1} \mid y_{0}\right) \dot{x}\left(t_{2} \mid y_{0}\right)\right\rangle=\left\langle U\left(y\left(t_{1} \mid y_{0}\right)\right) U\left(y\left(t_{2} \mid y_{0}\right)\right)\right\rangle+\left\langle u\left(t_{1}\right) u\left(t_{2}\right)\right\rangle,
$$

where the cross terms are zero $(\langle U u\rangle=0)$ since the random velocities $u$ are uncorrelated with the imposed velocity $U$. Using the results in $\S 3$, e.g. (3.4), $\left\langle u\left(t_{1}\right) u\left(t_{2}\right)\right\rangle=\overline{u^{2}} \exp \left(-\left|t_{2}-t_{1}\right| / \tau_{L}\right)$, only the shear-induced autocovariance, the first term on the right-hand side of (4.10), requires calculation. This term is the expected value of the product of the imposed velocity $U(y)$ that particles released from $y_{0}$ feel at $t=t_{1}$ and the imposed velocity felt at $t=t_{2}$. This term is calculated analogously to the mean Lagrangian longitudinal velocity but requires the j.p.d.f. of first and second displacements (derived in §3). In the bounded domain the shear-induced 
autocovariance is found from

$$
\left\langle U\left(y\left(t_{1} \mid y_{0}\right)\right) U\left(y\left(t_{2} \mid y_{0}\right)\right)\right\rangle=\int_{0}^{L} \int_{0}^{L} U\left(y_{1}\right) U\left(y_{2}\right) p_{12}\left(y_{2}, t_{2}, y_{1}, t_{1} \mid y_{0}\right) \mathrm{d} y_{2} \mathrm{~d} y_{1},
$$

where $p_{12}\left(y_{2}, t_{2}, y_{1}, t_{1} \mid y_{0}\right)$ is the probability density of finding a particle at $y_{2}\left(t=t_{2}\right)$ and at $y_{1}\left(t=t_{1}\right)$ given that the particle was originally at $y_{0}(t=0)$. As mentioned previously, unlike the readily determined $\tau_{L}=0$ bounded domain j.p.d.f. $p_{12}$ (Van Den Broeck 1982), the bounded domain j.p.d.f. $p_{12}$ for $\tau_{L} \neq 0$ is unknown. However, using the Haber \& Mauri (1988) method of expanding the imposed velocity in a cosine series, the transverse domain is periodically extended to infinity and

$$
\begin{aligned}
\left\langle U\left(y\left(t_{1} \mid y_{0}\right)\right) U\left(y\left(t_{2} \mid y_{0}\right)\right)\right\rangle= & \int_{-\infty}^{\infty} \int_{-\infty}^{\infty} \sum_{n=0}^{\infty} \sum_{m=0}^{\infty} U_{n} U_{m} \cos \left(\gamma_{n} y_{1}\right) \cos \left(\gamma_{m} y_{2}\right) \\
& \times \mathscr{P}_{12}\left(y_{2}, t_{2}, y_{1}, t_{1} \mid y_{0}\right) \mathrm{d} y_{2} \mathrm{~d} y_{1},
\end{aligned}
$$

where $\mathscr{P}_{12}\left(y_{2}, t_{2}, y_{1}, t_{1} \mid y_{0}\right)$ is the j.p.d.f. of displacements for particles originally at $y_{0}$ in the infinite $y$ domain. Again, transverse particle diffusion is homogeneous such that $\Delta y_{2}=y_{2}-y_{1}$ and $\Delta y_{1}=y_{1}-y_{0}$, thus $\mathscr{P}_{12}$ is the j.p.d.f. introduced earlier

$$
\mathscr{P}_{12}\left(y_{2}, t_{2}, y_{1}, t_{1} \mid y_{0}\right)=P_{12}\left(y_{2}-y_{1}, t_{2}, y_{1}-y_{0}, t_{1}\right) \text {, }
$$

where $P_{12}$ is given by (3.16). Expanding the sums in (4.12) results in

$$
\begin{aligned}
\left\langle U\left(y\left(t_{1} \mid y_{0}\right)\right) U\left(y\left(t_{2} \mid y_{0}\right)\right)\right\rangle= & \int_{-\infty}^{\infty} \int_{-\infty}^{\infty}\left\{U_{0}^{2}+U_{0} \sum_{n=1}^{\infty} U_{n}\left[\cos \left(\gamma_{n} y_{1}\right)+\cos \left(\gamma_{n} y_{2}\right)\right]\right. \\
& \left.+\sum_{n=1}^{\infty} \sum_{m=1}^{\infty} U_{m} U_{n} \cos \left(\gamma_{n} y_{1}\right) \cos \left(\gamma_{m} y_{2}\right)\right\} \\
& \times P_{12}\left(y_{2}-y_{1}, t_{2}, y_{1}-y_{0}, t_{1}\right) \mathrm{d} y_{2} \mathrm{~d} y_{1}
\end{aligned}
$$

and evaluating the integrals gives the initial-position-dependent shear-induced autocovariance

$$
\begin{aligned}
& \left\langle U\left(y\left(t_{1} \mid y_{0}\right)\right) U\left(y\left(t_{2} \mid y_{0}\right)\right)\right\rangle=U_{0}^{2}+U_{0} \sum_{n=1}^{\infty} U_{n} \cos \left(\gamma_{n} y_{0}\right)\left\{\mathrm{e}^{-\frac{1}{2} \sigma_{1}^{2} \gamma_{n}^{2}}+\mathrm{e}^{-\frac{1}{2}\left(\sigma_{1}^{2}+\sigma_{2}^{2}+2 \sigma_{12}^{2}\right) \gamma_{n}^{2}}\right\} \\
& +\sum_{m=1}^{\infty} \sum_{n=1}^{\infty} \frac{U_{m} U_{n}}{2} \mathrm{e}^{-\frac{1}{2}\left(\sigma_{2}^{2}-\left(\sigma_{12}^{4} / \sigma_{1}^{2}\right)\right) \gamma_{m}^{2}}\left\{\mathrm{e}^{-\frac{1}{2} \sigma_{1}^{2}\left(\gamma_{m}\left(1+\left(\sigma_{12}^{2} / \sigma_{1}^{2}\right)\right)+\gamma_{n}\right)^{2}} \cos \left[\left(\gamma_{m}+\gamma_{n}\right) y_{0}\right]\right. \\
& \left.+\mathrm{e}^{-\frac{1}{2} \sigma_{1}^{2}\left(\gamma_{m}\left(1+\left(\sigma_{12}^{2} / \sigma_{1}^{2}\right)\right)-\gamma_{n}\right)^{2}} \cos \left[\left(\gamma_{m}-\gamma_{n}\right) y_{0}\right]\right\},
\end{aligned}
$$

which is the non-zero Lagrangian time scale extension of equation (27) of Haber \& Mauri (1988). Note that this periodic velocity extension method only works if the infinite domain displacement p.d.f.s (and j.p.d.f.s) are independent of position, and can be written as functions of the displacements (i.e. $y_{2}-y_{1}$ ) only. Hence, unlike the bounded domain p.d.f. method of Van Den Broeck (1982), the method used here is not applicable for 3D pipe flow, because the (semi-)infinite domain p.d.f. for radial displacements depends explicitly on the particle's initial radial position $r_{0}$, and not radial displacements. Thus, radial displacements for particles which leave the bounded domain, which ought to be reflected, would be incorrectly represented in the infinite 
domain p.d.f. method. Hence, the infinite domain p.d.f. method (Haber \& Mauri 1988) used here is limited to certain geometries.

\subsection{The shear-induced diffusivity}

Like the effective transverse diffusivity $D_{y}$, the effective longitudinal diffusivity $D_{x}$ is the longitudinal displacement variance rate of change, namely

$$
D_{x}\left(t \mid y_{0}\right) \equiv \frac{1}{2} \frac{\mathrm{d}}{\mathrm{d} t}\left(\left\langle\left[x\left(t \mid y_{0}\right)\right]^{2}\right\rangle-\left\langle x\left(t \mid y_{0}\right)\right\rangle^{2}\right),
$$

which can be written in terms of velocity autocovariance integrals

$$
\begin{aligned}
D_{x}\left(t \mid y_{0}\right)= & \int_{0}^{t}\left\langle\dot{x}\left(t_{1} \mid y_{0}\right) \dot{x}\left(t \mid y_{0}\right)\right\rangle \mathrm{d} t_{1}-\left\langle\dot{x}\left(t \mid y_{0}\right)\right\rangle \int_{0}^{t}\left\langle\dot{x}\left(t_{1} \mid y_{0}\right)\right\rangle \mathrm{d} t_{1} \quad \text { which is } \\
= & \int_{0}^{t}[\underbrace{\left\langle\left\langle u\left(t_{1}\right) u(t)\right\rangle\right.}_{\text {(i) }}+\underbrace{\left\langle U\left(y\left(t_{1} \mid y_{0}\right)\right) U\left(y\left(t \mid y_{0}\right)\right)\right\rangle}_{\text {(ii) }} \\
& -\underbrace{\left\langle\dot{x}\left(t \mid y_{0}\right)\right\rangle\left\langle\dot{x}\left(t_{1} \mid y_{0}\right)\right\rangle}_{\text {(iii) }}] \mathrm{d} t_{1} .
\end{aligned}
$$

The effective diffusivity involves three terms: (i) the autocovariance of random longitudinal velocities; (ii) the shear-induced longitudinal velocity autocovariance; and (iii) a particle's mean longitudinal velocity. Term (i) is due to random longitudinal motions and given by

$$
\int_{0}^{t}\left\langle u\left(t_{1}\right) u(t)\right\rangle \mathrm{d} t_{1}=\int_{0}^{t} \overline{u^{2}} \exp \left(-\left|t-t_{1}\right| / \tau_{L}\right) \mathrm{d} t_{1}=\kappa\left(1-\mathrm{e}^{-t / \tau_{L}}\right),
$$

whereas terms (ii) and (iii) are both shear-induced. Thus, the effective longitudinal diffusivity is

$$
D_{x}\left(t \mid y_{0}\right)=\kappa\left(1-\mathrm{e}^{-t / \tau_{L}}\right)+D_{S}\left(t \mid y_{0}\right),
$$

where $D_{S}\left(t \mid y_{0}\right)$ is the time-dependent shear-induced diffusivity, i.e. $D_{S}$ is terms (ii) + (iii) of (4.17). Using (4.15) in (4.17)(ii) and (4.9) in (4.17)(iii), the shear-induced diffusivity is

$$
\begin{aligned}
D_{S}\left(t \mid y_{0}\right)= & U_{0} \sum_{n=1}^{\infty} U_{n} \cos \left(\gamma_{n} y_{0}\right) \int_{0}^{t} \mathrm{e}^{-\frac{1}{2}\left[\varsigma_{1}^{2}+\varsigma_{2}^{2}+2 \varsigma_{12}^{2}\right] \gamma_{n}^{2}} \mathrm{~d} t_{1}-U_{0}^{2} t \sum_{n=1}^{\infty} \cos \left(\gamma_{n} y_{0}\right) \mathrm{e}^{-\frac{1}{2} \varsigma^{2} \gamma_{n}^{2}} \\
& +\sum_{m=1}^{\infty} \sum_{n=1}^{\infty} \frac{U_{m} U_{n}}{2} \int_{0}^{t} \mathrm{e}^{-\frac{1}{2}\left(\varsigma_{2}^{2}-\left(\varsigma_{12}^{4} / \varsigma_{1}^{2}\right)\right) \gamma_{m}^{2}}\left\{\mathrm{e}^{-\frac{1}{2} \varsigma_{1}^{2}\left(\gamma_{m}\left(1+\left(\varsigma_{12}^{2} / \varsigma_{1}^{2}\right)\right)+\gamma_{n}\right)^{2}}\right. \\
& \left.\times \cos \left[\left(\gamma_{m}+\gamma_{n}\right) y_{0}\right]+\mathrm{e}^{-\frac{1}{2} \varsigma_{1}^{2}\left(\gamma_{m}\left(1+\left(\varsigma_{12}^{2} / \varsigma_{1}^{2}\right)\right)-\gamma_{n}\right)^{2}} \cos \left[\left(\gamma_{m}-\gamma_{n}\right) y_{0}\right]\right\} \mathrm{d} t_{1} \\
& -\left[\sum_{n=1}^{\infty} U_{n} \cos \left(\gamma_{n} y_{0}\right) \mathrm{e}^{-\frac{1}{2} \varsigma^{2} \gamma_{n}^{2}}\right]\left[\sum_{n=1}^{\infty} U_{n} \cos \left(\gamma_{n} y_{0}\right) \int_{0}^{t} \mathrm{e}^{-\frac{1}{2} s_{1}^{2} \gamma_{n}^{2}} \mathrm{~d} t_{1}\right]
\end{aligned}
$$

where

$$
\varsigma_{1}^{2} \equiv \sigma_{1}^{2}\left(t_{1}\right), \quad \varsigma_{2}^{2} \equiv \sigma_{2}^{2}\left(t_{1}, t\right), \quad \varsigma_{12}^{2} \equiv \sigma_{12}^{2}\left(t_{1}, t\right) \quad \text { and } \quad \varsigma^{2} \equiv \sigma_{1}^{2}(t)
$$

The expression (4.20) describes the imposed shear velocity's affect on longitudinal particle spreading rates when the random velocities have a finite Lagrangian time scale. 
The shear-induced diffusivity $D_{S}\left(t \mid y_{0}\right)$ depends on time $t$, particle release location $y_{0}$, the velocity profile through $U_{n}$, the domain width $L$, the transverse dispersion properties $\kappa$ and the Lagrangian time scale $\tau_{L}$.

\section{Properties of the shear-induced diffusivity}

Properties of the shear-induced diffusivity $D_{S}$ are explored by first isolating the relevant parameters. The expression for $D_{S}(4.20)$ is for any square-integrable velocity profile. A parabolic velocity profile, $U(y)=6 U_{0} y(1-y / L) / L$, is chosen in order to compare the effects of $\tau_{L} \neq 0$ with previous results for $\tau_{L}=0$ (e.g. Taylor 1953; Haber \& Mauri 1988, and others), even though a turbulent $\left(\tau_{L} \neq 0\right)$ flow will not have such a laminar velocity profile. The Fourier coefficients for this parabolic $U(y)$ are

$$
U_{n}= \begin{cases}U_{0} & \text { for } n=0 \\ -12 U_{0} \frac{1+(-1)^{n}}{n^{2} \pi^{2}} & \text { for } n \geqslant 1,\end{cases}
$$

which will be used subsequently whenever a specific velocity profile is needed.

It is also possible to remove some of the other dependencies by nondimensionalizing the shear-induced diffusivity $D_{S}$. By scaling $D_{S}$ in (4.20) by $U_{0}^{2} \tau_{D}$ where $\tau_{D}=L^{2} / \kappa$, the resulting expression depends on time $t$ and two parameters: $\tau_{L} / \tau_{D}$ and $y_{0} / L$. As the effect of $\tau_{L}$ on $D_{S}$ is of primary interest, $\kappa$ (recall that $\kappa=\overline{v^{2}} \tau_{L}$ ) will be kept fixed when varying $\tau_{L}$ by changing $\overline{v^{2}}$ accordingly. Hence, $\kappa$, and therefore $\tau_{D}$, can be considered constants as the effect of varying $\tau_{L} / \tau_{D}$ and $y_{0} / L$ on the time dependence and long-time limit of $D_{S}$ will be investigated. Although all of these dependencies will be addressed, it is instructive to first remove the $y_{0}$ dependence by considering the special case where particles are initially uniformly distributed.

\subsection{The uniform initial distribution}

The dependence of initial particle position $y_{0}$ is removed by considering particles which are initially uniformly distributed across the domain. The shear-induced diffusivity for an initially uniform particle distribution is a considerable simplification of the point-release version (4.20). To derive $D_{S}$ for initially uniformly distributed particles, $\langle\dot{x}(t)\rangle$ and $\left\langle\dot{x}\left(t_{1}\right) \dot{x}\left(t_{2}\right)\right\rangle$ need to be determined for initially uniformly distributed particles with p.d.f. $P_{0}(y)=1 / L$. These two statistics are readily obtained for any $P_{0}(y)$ from the previous point release statistics, e.g. (4.9) and (4.15), since the previous point release versions are like Green's functions, hence

$$
\left\langle\dot{x}\left(t \mid P_{0}\right)\right\rangle=\int_{0}^{L}\left\langle\dot{x}\left(t \mid y_{0}\right)\right\rangle P_{0}\left(y_{0}\right) \mathrm{d} y_{0}
$$

and

$$
\left\langle\dot{x}\left(t_{1} \mid P_{0}\right) \dot{x}\left(t_{2} \mid P_{0}\right)\right\rangle=\int_{0}^{L}\left\langle\dot{x}\left(t_{1} \mid y_{0}\right) \dot{x}\left(t_{2} \mid y_{0}\right)\right\rangle P_{0}\left(y_{0}\right) \mathrm{d} y_{0} .
$$

For an initially uniform particles distribution $P_{0}=1 / L$ and $(5.2 a)$ is the integral of (4.9) over $y_{0}$. Hence, the uniform-release mean Lagrangian velocity is the imposed transverse mean velocity $\langle\dot{x}(t \mid 1 / L)\rangle=U_{0}$ as $\int_{0}^{L} \cos \left(\gamma_{n} y_{0}\right) \mathrm{d} y_{0}=0$ for all $n \geqslant 1$. Similarly, for the shear-induced component of $\left\langle\dot{x}\left(t_{1} \mid 1 / L\right) \dot{x}\left(t_{2} \mid 1 / L\right)\right\rangle$, the integral of (4.15) over $y_{0}$ results in only two non-zero terms, the $U_{0}^{2}$ term and the term with $\cos \left[\left(\gamma_{m}-\gamma_{n}\right) y_{0}\right]$ for $m=n$ since $\cos \left[\left(\gamma_{m}-\gamma_{n}\right) y_{0}\right]=1$ in this case. 
Using these results, the shear-induced diffusivity calculated from (4.17)(ii) and (4.17)(iii) for an initially uniform distribution of particles is

$$
D_{S}(t)=\sum_{n=1}^{\infty} \frac{U_{n}^{2}}{2} \int_{0}^{t} \mathrm{e}^{-\frac{1}{2} \varsigma_{2}^{2} \gamma_{n}^{2}} \mathrm{~d} t_{1}
$$

or

$$
=\sum_{n=1}^{\infty} \frac{U_{n}^{2}}{2} \int_{0}^{t} \mathrm{e}^{-\frac{1}{2} s_{1}^{2} \gamma_{n}^{2}} \mathrm{~d} t_{1}
$$

where the last expression (5.3b) is found by changing variables, $t-t_{1} \rightarrow t_{1}$, in (5.3a). For brevity, the conditional notation is omitted and $D_{S}(t \mid 1 / L)=D_{S}(t)$. Properties of $D_{S}$ for a initially uniformly distributed particles are now investigated.

\subsection{The large-time shear-induced diffusivity}

Shear dispersion was first investigated for large times and $\tau_{L}=0$, resulting in the classic 'Taylor' asymptotic diffusivity $D_{T}^{\infty}=U_{0}^{2} \tau_{D} / 210=U_{0}^{2} L^{2} / 210 \kappa$ (Taylor 1953). Here, the analogous result but with $\tau_{L} \neq 0$ is examined. The large-time shear-induced diffusivity is defined as

$$
D_{S}^{\infty}=\lim _{t \rightarrow \infty} D_{S}(t),
$$

where $D_{S}(t)$ refers to the shear-induced diffusivity for an initially uniform particle distribution. Note that it is not necessary to define $D_{S}^{\infty}$ in terms of an initially uniform distribution as $D_{S}^{\infty}$ is independent of the initial particle distribution since for large $t$ particles are uniformly distributed in the transverse direction regardless of the initial distribution. Moreover, the large-time limit of the shear-induced diffusivity for particles released from any $y_{0}$ (4.20) indicates that all terms in (4.20) containing $\cos \left(y_{0}\right)$ are exponentially small, thus only the $m=n$ term in the double sum remains, the same term that remains for the uniform initial distribution $D_{S}(t)$ (5.3). Thus, the large-time shear-induced diffusivity $D_{S}^{\infty}$ is (from $(5.3 b)$ )

$$
D_{S}^{\infty}=\sum_{n=1}^{\infty} \frac{U_{n}^{2}}{2} \int_{0}^{\infty} \mathrm{e}^{-\frac{1}{2} s_{1}^{2} \gamma_{n}^{2}} \mathrm{~d} t_{1},
$$

which has no closed-form solution due to $\zeta_{1}^{2}=2 \kappa\left(t_{1}+\tau_{L} \exp \left(-t_{1} / \tau_{L}\right)-\tau_{L}\right)$ in the exponential. However, for each $n$, the integral in (5.5) can be calculated numerically and then summed over $n$ (an upper limit of $n=50$ is more than sufficient) to obtain $D_{S}^{\infty}$.

With the parabolic velocity profile Fourier coefficients $U_{n}$ (5.1), the effect of non-zero $\tau_{L}$ on $D_{S}^{\infty}$ is studied by examining the $D_{S}^{\infty} / D_{T}^{\infty}$ dependence on $\tau_{L} / \tau_{D}$, where $\tau_{D}=L^{2} / \kappa$ is the across-flow diffusive time scale (3.14). The ratio $D_{S}^{\infty} / D_{T}^{\infty}$ is significantly affected by non-zero $\tau_{L}$ (solid black curve in figure 4). At small $\tau_{L} / \tau_{D}=10^{-3}$, the large-time shear-induced diffusivity is nearly the Taylor diffusivity $\left(D_{S}^{\infty} / D_{T}^{\infty} \approx 1\right)$. However, at the relatively small $\tau_{L} / \tau_{D}=0.1$, the ratio $D_{S}^{\infty} / D_{T}^{\infty} \approx 3$ and is enhanced substantially. For a Lagrangian time scale equal to diffusive time $\left(\tau_{L} / \tau_{D}=1\right), D_{S}^{\infty} / D_{T}^{\infty} \approx 8$. Thus, for $\tau_{L} / \tau_{D}>0.01$, a non-zero Lagrangian time scale significantly $(>50 \%)$ enhances the large-time shear dispersion over that of the classic Taylor result. From Monte Carlo simulations of particle trajectories, this $\tau_{L} \neq 0$ enhancement was also found for sinusoidal shear flow $U(y)=\sin (y)$ (analogous to 


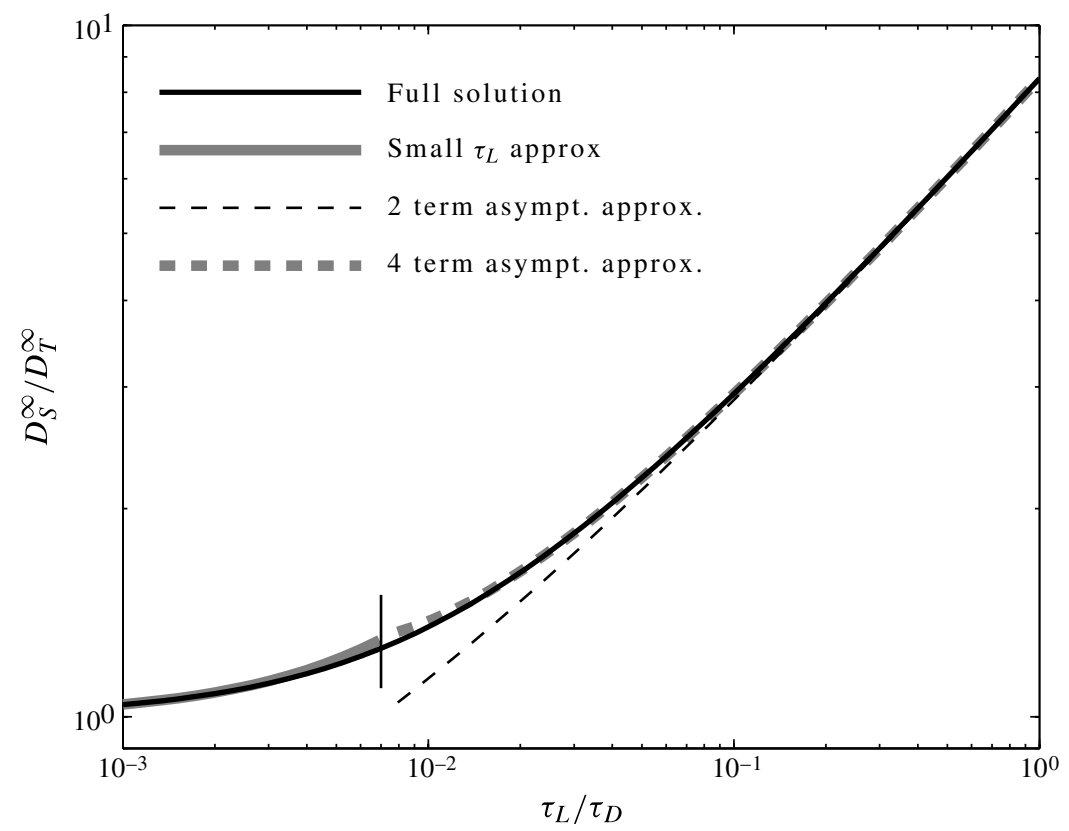

FIgURE 4. The ratio $D_{S}^{\infty} / D_{T}^{\infty}$ (the long-time shear-induced diffusivity to the Taylor diffusivity) versus $\tau_{L} / \tau_{D}$ (the ratio of the Lagrangian time scale to the diffusive time). The full solution (5.5) is indicated by the black curve, the small $\tau_{L}(6.7 a)$ and asymptotic $(6.7 b)$ approximations are indicated by the grey thick and grey thick dashed curves, respectively. The first two terms of the asymptotic approximation is indicated by the black thin dashed curve. The thin vertical line at $\tau_{L} / \tau_{D}=8 \times 10^{-3}$ indicates the boundary between the small $\tau_{L}$ and four-term large $\tau_{L}$ asymptotic approximations.

keeping only the $n=1$ term in (5.4)) by Pavliotis et al. (2009). Similarly, in numerical simulations of dispersion within a steady Taylor-Green flow, the effective diffusivity for $\tau_{L} \neq 0$ can be as large as 1000 times the $\tau_{L}=0$ value (Pavliotis \& Stuart 2005). The $D_{S}^{\infty} / D_{T}^{\infty}$ dependence on $\tau_{L}$ is further examined with asymptotic analysis in $\$ 6.2$.

\subsection{Time dependence of $D_{S}$}

After considering the large-time limit $D_{S}^{\infty}$ which is independent of time and the initial particle distribution, the dependence of $D_{S}$ on initial position, time and Lagrangian time scale is considered. First the time dependence for different initial release distributions is examined and then the effect of $\tau_{L} / \tau_{D}$ on the time dependence for initially uniformly distributed particles is investigated.

\subsubsection{Effect of initial distribution}

The effect of the initial particle position on the $D_{S}\left(t \mid y_{0}\right)$ time dependence is considered by varying $y_{0}$ and keeping $\tau_{L} / \tau_{D}$ fixed. For $\tau_{L}=0$, the effect of initial particle position has been considered (Latini \& Bernoff 2001; Camassa et al. 2010). The shear-induced diffusivity $D_{S}\left(t \mid y_{0}\right)$ is calculated for $y_{0}=L / 2$ and $y_{0}=L / 4$ with (4.20), and for an initially uniform particle distribution given by (5.3). The $D_{S}\left(t \mid y_{0}\right)$ are calculated numerically in the same manner as $D_{S}^{\infty}$ in $\S 5.2$. However, now integrals of the form $\int_{0}^{t} \exp \left(-\gamma_{n}^{2} S_{1}^{2} / 2\right) \mathrm{d} t_{1}$ are calculated numerically for all $t$, with $U_{n}$ given by (5.1) and a maximum $n=50$. Stochastic numerical simulations of the Langevin equations (2.1) in a bounded domain were performed for $10^{6}$ particle trajectories 


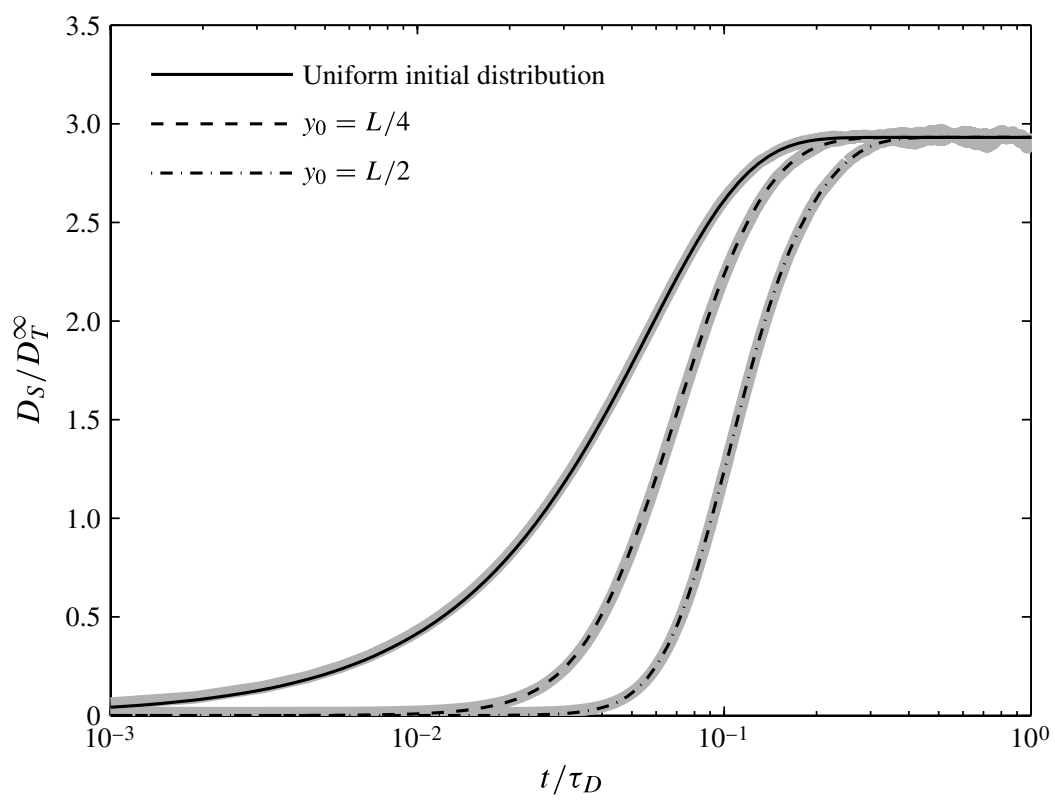

FIGURE 5. The shear-induced diffusivity (4.20) normalized by the Taylor diffusivity $D_{S}\left(t \mid y_{0}\right) / D_{T}^{\infty}$ versus $t / \tau_{D}$ with $\tau_{L} / \tau_{D}=0.1$ for three different initial release locations (black curves, see the legend). Thick grey curves, under the black curves, are from stochastic simulations of Langevin equations (2.1) in a bounded domain with particle reflection at the boundaries (i.e. $\eta_{B} \neq 0$ ) and particles released from the same locations.

released uniformly and from $y_{0}=L / 4$, and $y_{0}=L / 2$ with a Péclet number of $P e=U_{0} L / \kappa_{y}=10$ and a forward Euler time step $\Delta t / \tau_{L}=1 / 80$. In the simulations, particle reflection at the boundaries (i.e. $\eta_{B} \neq 0$ in $(2.1 b)$ ), is implemented as follows. At every time step, if a particle has left the domain $(y<0$ or $y>L)$, the particle's transverse location is reflected, $y \rightarrow 2 L-y$ for $y>L$ or $y \rightarrow-y$ for $y<0$, and the sign of the particle's transverse velocity is flipped $(v \rightarrow-v)$. As the imposed velocity is $U(y)$, numerical problems occurring when the Langevin equations are time stepped for large Pe using an Euler method were not observed (e.g. Pavliotis et al. 2009). The Lagrangian time scale is fixed at $\tau_{L} / \tau_{D}=0.1$, which results in a substantially increased $D_{S}^{\infty} / D_{T}^{\infty} \approx 3$ (e.g. figure 4).

The shear-induced diffusivity $D_{S}\left(t \mid y_{0}\right)$ time dependence for $y_{0}=L / 2, y_{0}=L / 4$, and for initially uniformly distributed particles is shown in figure 5 . The numerical particle simulations result in an effective diffusivity identical to the theoretical $D_{S}\left(t \mid y_{0}\right)$ (compare black curves and thick grey curves under them in figure 5), confirming the theory. The uniformly distributed release $D_{S}(t)$ has the shortest ramp-up time (solid black curve in figure 5) because uniformly distributed releases feel the entire velocity shear from the moment of release. For particles released at $y_{0}=L / 2, D_{S}\left(t \mid y_{0}\right)$ (black dashed-dot curve in figure 5) has the longest ramp-up time due to the initially zero velocity shear felt at $y_{0}=L / 2$. For particles released at $y_{0}=L / 4, D_{S}\left(t \mid y_{0}\right)$ (black dashed curve in figure 5) ramps up more rapidly than for $y_{0}=L / 2$ due to the non-zero velocity shear felt at $y_{0}=L / 4$. For $\tau_{L}=0$, the dependence of ramp-up time on the initial particle distribution, point release and uniform, can be derived (Camassa et al. 2010) and has similar release location dependence as for $\tau_{L} \neq 0$ (figure 5). In particular, the ramp-up time for an initially uniform distribution is shorter than for a 


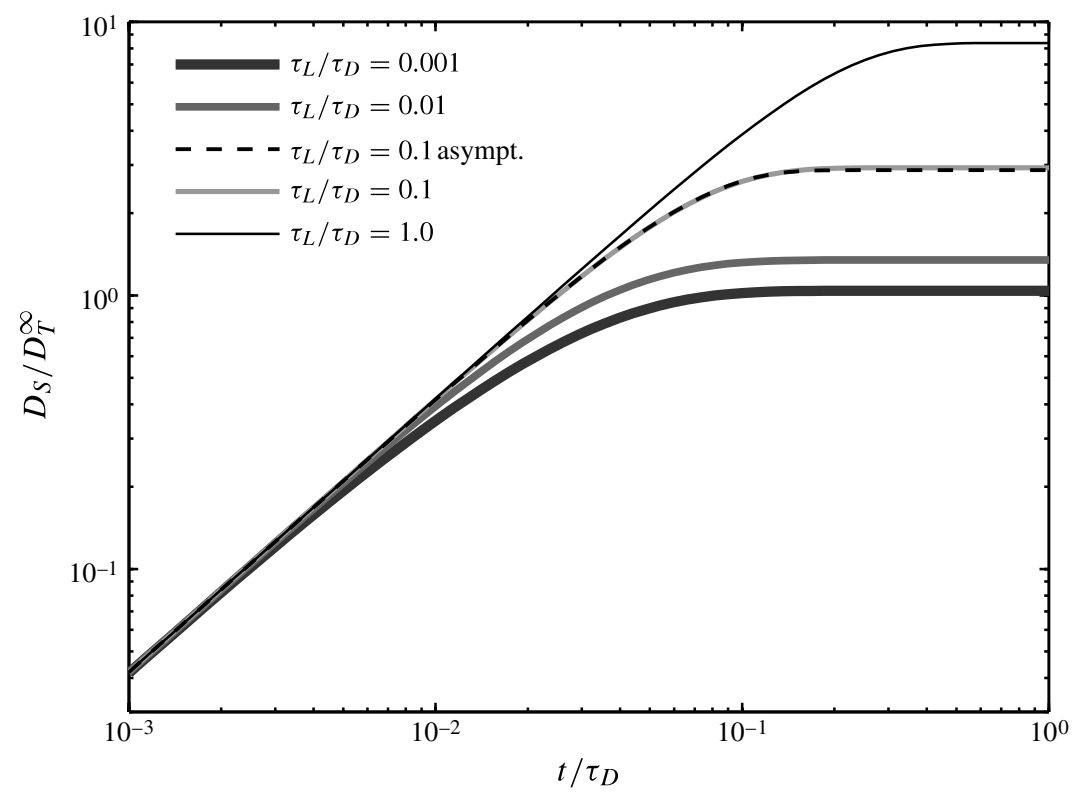

FIGURE 6 . The shear-induced diffusivity scaled by the Taylor diffusivity $D_{S}(t) / D_{T}^{\infty}$ versus $t / \tau_{D}$ for four different $\tau_{L} / \tau_{D}$ (see legend). Particles are initially uniformly distributed for all curves. The dashed curve is the asymptotic approximation (6.3).

point release. As demonstrated, the large-time shear-induced diffusivity $D_{S}^{\infty}$ does not depend upon the initial particle distribution and is larger than the $\tau_{L}=0$ Taylor result $D_{S}^{\infty} \approx 3 D_{T}^{\infty}$ (i.e. figure 4 ).

\subsubsection{Effect of the Lagrangian time scale}

To remove the dependence on $y_{0} / L$, the effect of $\tau_{L}$ on the shear-induced diffusivity time dependence is studied by considering an initially uniform particle distribution where $D_{S}$ is given by $(5.3 b)$. The time dependence of $D_{S}(t) / D_{T}^{\infty}$ is shown in figure 6 for various $\tau_{L} / \tau_{D}$, where, as before, the integral in $(5.3 b)$ is calculated numerically. As shown in figure 4, the large-time shear-induced diffusivity $D_{S}^{\infty}$ increases with increasing $\tau_{L}$. In addition, the time it takes $D_{S}(t)$ to reach its large-time value, the 'ramp-up time', increases with increasing $\tau_{L}$ (figure 6). In particular, for $\tau_{L} / \tau_{D}$ between $10^{-3}$ and 1 , the ramp-up time varies by approximately an order of magnitude (compare thick and thin black curves in figure 6). For initially uniformly distributed particles, the very small time behaviour $\left(t \ll \tau_{D}\right)$ of the shear-induced diffusivity is independent of $\tau_{L}$ (the curves collapse for $t / \tau_{d} \ll 1$ to a single line in figure 6). This is demonstrated by assuming that $\exp \left(-\gamma_{n}^{2} S_{1}^{2} / 2\right) \approx 1$ in $(5.3 b)$ and results in the small-time $\left(t / \tau_{D} \ll 1\right) D_{S}(t)$ given by $D_{S}(t) \approx U_{0}^{2} t / 5$ for all $\tau_{L} / \tau_{D}$. The dependence of the ramp-up time on $\tau_{L}$ will be clarified in the next section by considering the asymptotic approximation to the shear-induced diffusivity for an initially uniform particle distribution.

\section{Asymptotic analysis of the shear-induced diffusivity}

Asymptotic analysis of $D_{S}(t)$ provides insight into time dependence and large-time limit of $D_{S}(t)$ and its dependence upon $\tau_{L} / \tau_{D}$, bypassing the need for numerical integration. The asymptotic time dependence of $D_{S}(t)$ is investigated first. 


\subsection{Asymptotic time dependence}

This analysis requires the asymptotic approximation of the integral $\int_{0}^{t} \exp \left(-\gamma_{n}^{2} S_{1}^{2} / 2\right) \mathrm{d} t_{1}$ in $(5.3 b)$ for finite $\tau_{L}$ since a closed-form solution does not exist. Using Taylor's method of asymptotic integration (see appendix B), in the asymptotic limit of large $\tau_{L}$, the integral becomes

$$
\begin{aligned}
\int_{0}^{t} \mathrm{e}^{-\frac{1}{2} s_{1}^{2} \gamma_{n}^{2}} \mathrm{~d} t_{1} \sim & \frac{\tau_{S}}{n \sqrt{2 \pi}} \operatorname{erf}\left(\frac{n \pi t}{\sqrt{2} \tau_{S}}\right)+\frac{1}{3 n^{2} \pi^{2}} \alpha \\
& -\mathrm{e}^{-\frac{1}{2} \pi^{2} n^{2}\left(\frac{t}{\tau_{S}}\right)^{2}}\left[\frac{1}{3 n^{2} \pi^{2}}+\frac{1}{6}\left(\frac{t}{\tau_{S}}\right)^{2}\right] \alpha+O\left(\alpha^{2}\right),
\end{aligned}
$$

where a new time scale $\tau_{S}$ and ratio $\alpha$ are defined as

$$
\tau_{S}=\sqrt{\tau_{L} \tau_{D}} \text { and } \alpha=\sqrt{\tau_{D} / \tau_{L}} .
$$

Plugging this into (5.3b) results in the asymptotic approximation of the shear-induced time-dependent diffusivity for an initially uniform particle distribution

$$
\begin{aligned}
D_{S}(t) \sim & \sum_{n=1}^{\infty} \frac{U_{n}^{2} \tau_{S}}{2}\left\{\frac{1}{n \sqrt{2 \pi}} \operatorname{erf}\left(\frac{n \pi t}{\sqrt{2} \tau_{S}}\right)+\frac{1}{3 n^{2} \pi^{2}} \alpha\right. \\
& \left.-\mathrm{e}^{-\frac{1}{2} \pi^{2} n^{2}\left(\frac{t}{\tau_{S}}\right)^{2}}\left[\frac{1}{3 n^{2} \pi^{2}}+\frac{1}{6}\left(\frac{t}{\tau_{S}}\right)^{2}\right] \alpha\right\}+O\left(\alpha^{2}\right) .
\end{aligned}
$$

Although the error terms are proportional to $\alpha=\left(\tau_{D} / \tau_{L}\right)^{1 / 2}$, this $O(\alpha)$ asymptotic result is nearly the full result, over four orders of magnitude in $t / \tau_{D}$, even for a 'small' Lagrangian time scale (the dashed line in figure 6 is $(6.3)$ for $\tau_{L} / \tau_{D}=0.1$ ). Hence, although (6.3) is better for larger $\tau_{L}$, it is still quite good for relatively small $\tau_{L}$.

It is of interest to compare this asymptotic result to the very small $\tau_{L}(\alpha \gg 1)$ version of the shear-induced diffusivity

$$
D_{S}(t) \sim \sum_{n=1}^{\infty} \frac{U_{n}^{2} \tau_{D}}{2}\left[\frac{1}{n^{2} \pi^{2}}+\frac{1}{\alpha^{2}}\right]\left[1-\mathrm{e}^{-n^{2} \pi^{2} t / \tau_{D}}\right]+O\left(1 / \alpha^{4}\right) \quad \text { for } \alpha \gg 1,
$$

derived using $\gamma_{n}^{2} \varsigma_{1}^{2} / 2 \approx n^{2} \pi^{2}\left(t_{1}+\tau_{L}\right) / \tau_{D}+$ e.s.t. in (5.3b), where 'e.s.t.' denotes exponentially small terms. The leading-order term in (6.4), proportional to $1 /(n \pi)^{2}$, is the time-dependent Taylor diffusivity $D_{T}(t)$ (e.g. Barton 1983). By comparing the leading-order term of the very small $\tau_{L}$ shear-induced diffusivity (6.4) to the leadingorder term of the asymptotic shear-induced diffusivity (6.3), an important difference between non-zero and zero $\tau_{L}$ dispersion is evident. For $\tau_{L} \neq 0$, the ramp-up time is governed by $\operatorname{erf}\left(t / \tau_{S}\right)$ with time scale $\tau_{S}$ whereas for $\tau_{L}=0$, ramp-up is governed by $1-\exp \left(-t / \tau_{D}\right)$ with time scale $\tau_{D}$. Thus, for non-zero Lagrangian time-scale dispersion (as long as $\left.\tau_{L}>0.01 \tau_{D}\right), \tau_{S}$ is the natural time scale for the shear-induced diffusivity.

\subsection{Asymptotic large-time shear-induced diffusivity}

The asymptotic large-time shear-induced diffusivity is the large-time limit of the results above, thus it is dictated by the behaviour of $\int_{0}^{\infty} \exp \left(-\gamma_{n}^{2} \varsigma_{1}^{2} / 2\right) \mathrm{d} t_{1}$. Similar to the asymptotic time-dependent shear-induced $D_{S}(t)$ above, there are two regimes to 
consider: the small and large $\tau_{L}$ limits. For small $\tau_{L}$, the integral is

$$
\int_{0}^{\infty} \mathrm{e}^{-\frac{1}{2} s_{1}^{2} \gamma_{n}^{2}} \mathrm{~d} t_{1} \sim \frac{\tau_{D}}{n^{2} \pi^{2}}\left[1+n^{2} \pi^{2} \alpha^{-2}+\frac{n^{4} \pi^{4}}{2} \alpha^{-4}+O\left(\alpha^{-6}\right)\right] \text { for } \alpha \gg 1
$$

and at leading order is $\sim \tau_{D}$ with a $\tau_{L}$ correction at next order. For large $\tau_{L}$, the asymptotic approximation to the integral (derived in appendix B) is

$$
\begin{aligned}
& \int_{0}^{\infty} \mathrm{e}^{-\frac{1}{2} s_{1}^{2} \gamma_{n}^{2}} \mathrm{~d} t_{1} \sim \frac{\tau_{S}}{n \sqrt{2 \pi}}\left[1+\frac{\sqrt{2}}{3 n \pi^{3 / 2}} \alpha+\frac{1}{12 n^{2}} \alpha^{2}+\frac{4 \sqrt{2}}{135 n^{3} \pi^{7 / 2}} \alpha^{3}+O\left(\alpha^{4}\right)\right] \\
& \quad \text { for } \alpha \ll 1,
\end{aligned}
$$

which at leading order is $\sim \tau_{S}$ with a $\tau_{D}$ contribution at next order. This is considerably different than the $\sim \tau_{D}$ leading-order behaviour for small $\tau_{L} / \tau_{D}$. Plugging these integrals into (5.5) and performing the sums for $U_{n}$ given by (5.1) results in the large-time shear-induced diffusivity (scaled by the Taylor diffusivity)

$$
\frac{D_{S}^{\infty}}{D_{T}^{\infty}} \sim \begin{cases}1+42 \frac{1}{\alpha^{2}}+1260 \frac{1}{\alpha^{4}}+O\left(1 / \alpha^{6}\right) & \text { for } \tau_{L} \ll \tau_{D} \\ \frac{945 \sqrt{2} \zeta(5)}{\pi^{9 / 2}} \frac{1}{\alpha}+\frac{1}{3}+\frac{315 \sqrt{2} \zeta(7)}{16 \pi^{13 / 2}} \alpha+\frac{\alpha^{2}}{1350}+O\left(\alpha^{3}\right) & \text { for } \tau_{L} \gg \tau_{D}\end{cases}
$$

where $\zeta(x)=\sum_{n=1}^{\infty} 1 / n^{x}$. For small $\tau_{L}, D_{S}^{\infty}$ is perturbed from the Taylor value $D_{T}^{\infty}$ by a term proportional to $\tau_{L} / \tau_{D}$ and for $\tau_{L}<0.007 \tau_{D}$, this small $\tau_{L}$ approximation to $D_{S}^{\infty}$ (thick grey line in figure 4) is very close to the full solution. For finite $\tau_{L}$, the asymptotic large-time shear-induced diffusivity is at leading order given by

$$
D_{S}^{\infty} \sim 0.132 U_{0}^{2} \tau_{S}
$$

when all constants are evaluated. Thus, at leading order, $\tau_{S}=\sqrt{\tau_{D} \tau_{L}}$ is the time scale that determines the large-time limit $D_{S}^{\infty}$. Moreover, for non-zero $\tau_{L}$, the large-time shear-induced diffusivity $D_{S}^{\infty}$ depends upon $\sqrt{\tau_{L}}$. This leading-order dependency of $D_{S}^{\infty}$ on $\tau_{L}$ was also found for sinusoidal shear flow (Pavliotis et al. 2009).

The first two and four terms of $(6.7 b)$ reasonably reproduce the $D_{S}^{\infty} / D_{T}^{\infty}$ (dashed curves in figure 4). The two-term approximation is close to the full result for approximately $\tau_{L} / \tau_{D}>8 \times 10^{-2}$ (thin dashed curve in figure 4) whereas the four-term approximation is close to full result for approximately $\tau_{L} / \tau_{D}>7 \times 10^{-3}$ (thick dashed curve in figure 4). Although only formally valid for small $\alpha$, the four-term asymptotic approximation $(6.7 b)$ is accurate even for relatively large $\alpha$, in this case it is good for $\alpha<12$.

\subsection{The leading-order self-similar diffusivity}

At leading order, the normalized (by $D_{S}^{\infty}$ ) shear-induced diffusivity $D_{S}(t) / D_{S}^{\infty}$ for uniformly distributed particle release is

$$
D_{S}(t) / D_{S}^{\infty} \sim \sum_{n=1}^{\infty} \frac{U_{n}^{2}}{n} \operatorname{erf}\left(\frac{n \pi t}{\sqrt{2} \tau_{S}}\right) / \sum_{n=1}^{\infty} \frac{U_{n}^{2}}{n},
$$

which is a function of only $t / \tau_{S}$. Hence, for any moderately sized $\tau_{L}$, the normalized shear-induced diffusivity collapses onto a single function. The (non-asymptotic) numerically integrated $D_{S}(t) / D_{S}^{\infty},(5.3 b)$ divided by (5.5), is shown in figure 7 as 


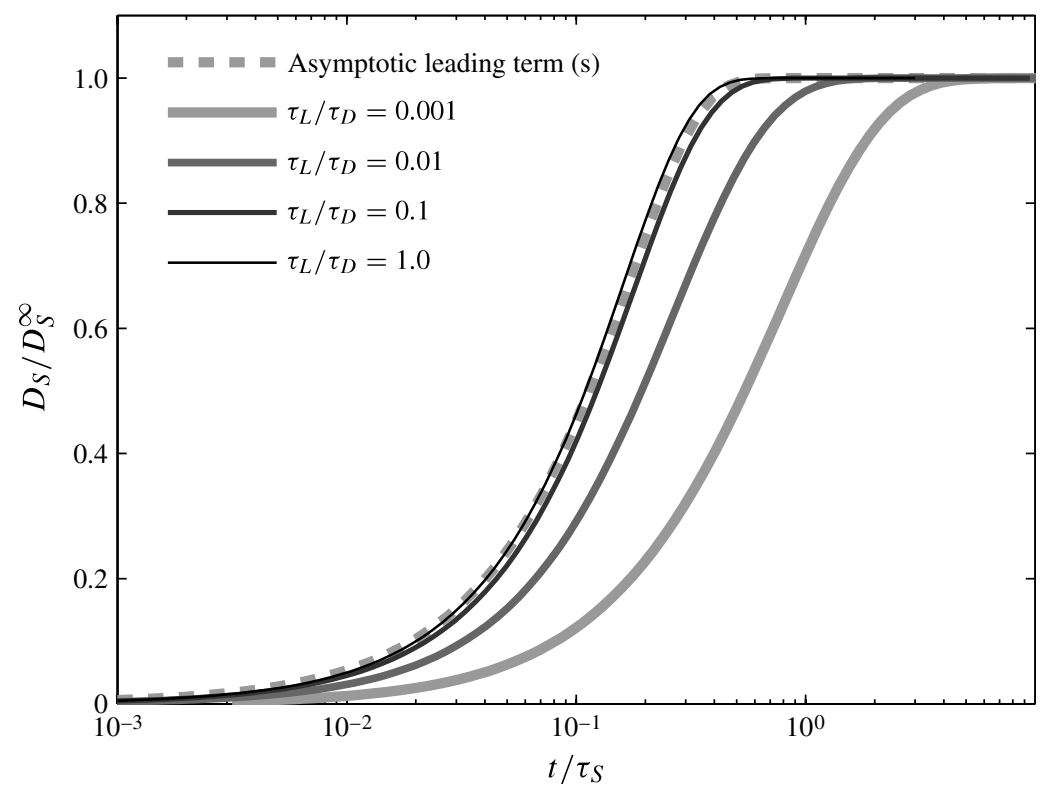

FIGURE 7. The shear-induced diffusivity scaled by the large-time shear-induced diffusivity $D_{S}(t) / D_{S}^{\infty}$ versus $t / \tau_{S}$ where $\tau_{S}=\left(\tau_{D} \tau_{L}\right)^{1 / 2}$ for four $\tau_{L} / \tau_{D}$ (see the legend) with initially uniformly distributed particles. The dashed line is constructed using the leading-order term of the asymptotic $D_{S}(t)$ and the asymptotic $D_{S}^{\infty}$, i.e. (6.9).

a function of $t / \tau_{S}$ for various $\tau_{L} / \tau_{D}$ (the solid curves in figure 7 are the same curves in figure 6 but with the $x$ - and $y$-axes rescaled). For $\tau_{L} / \tau_{D} \geqslant 0.1, D_{S}(t) / D_{S}^{\infty}$ collapses onto a single function of $t / \tau_{S}$ given by (6.9) (see the grey dashed curve in figure 7). At small $\tau_{L} / \tau_{D} \leqslant 0.01, D_{S}(t) / D_{S}^{\infty}$ does not collapse onto a single function of $t / \tau_{S}$ (solid grey curves in figure 7). This is not surprising since this is within the $\tau_{L} / \tau_{D}$ range where the two-term asymptotic approximation, which is better than the one-term approximation used in (6.9), for $D_{S}^{\infty}$ is no longer valid (figure 4).

\section{Discussion}

\subsection{The time scale for shear dispersion}

As shown, the large-time shear-induced diffusivity is given by $D_{S}^{\infty}=C U_{0}^{2} \tau$ where $C$ is a constant and $\tau$ is a time scale: for $\tau_{L} \ll \tau_{D}, \tau=\tau_{D}$; and for large $\tau_{L}\left(\tau_{L} / \tau_{D}>0.1\right)$, $\tau=\left(\tau_{D} \tau_{L}\right)^{1 / 2}$. By considering the time it takes a Lagrangian particle to cross the domain, the change in the time scale from $\tau_{D}$ to $\left(\tau_{D} \tau_{L}\right)^{1 / 2}$ can be anticipated. Solving $\sigma_{1}^{2}(\tau)=L^{2}$, where $\sigma_{1}^{2}$ is given by $(3.11 a)$, i.e.

$$
2 \kappa\left[\tau+\tau_{L} \mathrm{e}^{-\tau / \tau_{L}}-\tau_{L}\right]=L^{2},
$$

for $\tau$ gives the crossing time. As solving explicitly for $\tau$ in (7.1) is not possible, approximate methods are used. For small $\tau_{L}\left(\tau_{L} \ll \tau_{D}\right)$, the left-hand side of (7.1) becomes $2 \kappa \tau$ and the correct time scale $\tau=L^{2} / 2 \kappa$ or $\tau=\tau_{D} / 2$ is recovered. For large $\tau_{L}$, the left-hand side of (7.1) can be expanded in a Taylor series so that at leading order (7.1) becomes

$$
\frac{\kappa \tau^{2}}{\tau_{L}}=L^{2}
$$


and $\tau=\sqrt{\tau_{D} \tau_{L}}$. Hence, the time scale in the formula $D_{S}^{\infty}=C U_{0}^{2} \tau$ is the Lagrangian particle domain-crossing time.

Elegant and intuitive derivations of the shear-induced diffusivity scaling $D_{S} \propto U_{0}^{2} \tau$ are given by Taylor (1953) and Young \& Jones (1991). Both particle domain-crossing times regimes, $\tau=\left(\tau_{D} \tau_{L}\right)^{1 / 2}$ and $\tau=\tau_{D}$, can be anticipated on physical grounds. Consider a domain with a few persistent (i.e. large $\tau_{L}$ ) eddies with transverse velocity scale $\sqrt{\overline{v^{2}}}$ superimposed upon a mean longitudinal current. In this case, particles are effectively advected by the eddies across the domain and the transverse domain crossing time is $\tau=L / \sqrt{\overline{v^{2}}}=\left(\tau_{D} \tau_{L}\right)^{1 / 2}$. For a constant $\kappa$, as assumed here, $\left(\tau_{D} \tau_{L}\right)^{1 / 2}=\left(L^{2} \tau_{L} / \kappa\right)^{1 / 2}$, and the increase of $D_{S}^{\infty}$ with $\tau_{L}$ is anticipated. In contrast, for constant $\overline{v^{2}}$ rather than constant $\kappa$, the time scale $\tau=L / \sqrt{\overline{v^{2}}}$ is independent of $\tau_{L}$. For a transverse domain filled with many rapidly evolving eddies (small $\tau_{L}$ ), so that particles spread eddy diffusively, the appropriate transverse crossing time is Taylor's time scale $\tau=\tau_{D}=L^{2} / \kappa$.

\subsection{Relationship to turbulent dispersion in pipes and channels}

The shear-induced diffusivity $D_{S}$ derived here is for random non- $\delta$ correlated Lagrangian velocities superimposed upon a bounded, sheared mean flow. Although the random velocities and $\tau_{L}$ are often inhomogeneous in general turbulent flows, this $D_{S}^{\infty}$ can be approximately related to the turbulent longitudinal diffusivity found for wall-bounded turbulent shear flows. For both turbulent flows in pipes of width $L$ (Taylor 1954) or channels of depth $L$ (Elder 1959), the large-time longitudinal shearinduced diffusivity $D$ has been shown to be $D \propto u_{*} L$, where $u_{*}$ is the friction velocity. Using representative values for $\tau_{L}$ and r.m.s. transverse velocity fluctuation $\sqrt{\overline{v^{2}}}$ within an inhomogeneous turbulent flow, a representative value of the eddy diffusivity is $\kappa \approx \bar{v}^{2} \tau_{L}$ and the non-zero $\tau_{L}$ shear-induced large-time diffusivity given by (6.8) becomes $D_{S}^{\infty} \propto U_{0}^{2} L / \sqrt{\overline{v^{2}}}$. In the analysis here, $\sqrt{\overline{v^{2}}}$ was prescribed independently of the mean velocity $U_{0}$. However, in many turbulent settings the velocity fluctuations are related to the mean flow. In particular, for wall-bounded turbulent shear-flow (e.g. Tennekes \& Lumley 1972), the r.m.s. velocity fluctuations $\left(\sqrt{\overline{v^{2}}}\right)$ and $U_{0}$ are both proportional to the friction velocity $u_{*}$. Thus, $U_{0}^{2} / \sqrt{\overline{v^{2}}} \propto u_{*}$ yielding $D_{S}^{\infty} \propto u_{*} L$, consistent with the turbulent dispersion results of Taylor (1954) and Elder (1959).

\section{Conclusions}

Dispersion in a sheared flow (shear-dispersion) previously has been studied analytically for diffusion with zero Lagrangian time scale $\tau_{L}=0$ (i.e. molecular diffusion), and applied to a number of engineering and geophysical flow settings. However, various mean-sheared turbulent geophysical flows, including western boundary current extensions, the Antarctic Circumpolar Current, and surfzone alongshore currents, have a $\tau_{L}$ that is not very small relative to the diffusive time scale. To illustrate the role of non-zero $\tau_{L}$ in shear dispersion processes, shear dispersion in a two-dimensional bounded sheared mean flow for a diffusive process with a constant $\tau_{L} \neq 0$ was examined.

Analysis of the transverse (across-flow) particle statistics was performed in a unbounded periodic domain by extending the imposed longitudinal velocity in a cos series, allowing estimation of the longitudinal shear-induced diffusivity. Although this method does not correctly reproduce the bounded transverse statistics, the classic 
longitudinal particle dispersion statistics in a bounded domain are recovered for $\tau_{L} \rightarrow 0$. To calculate the $\tau_{L} \neq 0$ longitudinal shear-induced diffusivity, the key analysis piece is the correlation of transverse particle displacements, induced by a finite Lagrangian time scale $\tau_{L}$, as a function of two subsequent displacement times. For $\tau_{L}=0$, the correlation is zero.

Using the j.p.d.f. of successive transverse particle displacements, and the imposed sheared velocity, the analytic expression for the time-dependent non-zero $\tau_{L}$ longitudinal shear-induced diffusivity $D_{S}(t)$ was derived for both a single transverse release location $y_{0}$ and a uniformly distributed particle release. The shear-induced diffusivity $D_{S}$ scaled by classic Taylor diffusivity is a function of time and depends upon non-dimensional parameters $\tau_{L} / \tau_{D}$ and release location $y_{0} / L$. The properties of the shear-induced diffusivity were investigated by numerically evaluating the expressions with the imposed classic parabolic velocity profile used in previous investigations.

At all $\tau_{L} / \tau_{D}$, the large-time $D_{S}^{\infty}$ is enhanced over the Taylor diffusivity $\left(D_{S}^{\infty} / D_{T}^{\infty} \geqslant 1\right)$ and increases with $\tau_{L} / \tau_{D}$. The enhancement is significant even for relatively small Lagrangian time scales, in particular for $\tau_{L} / \tau_{D}=0.1, D_{S}^{\infty} / D_{T}^{\infty} \approx 3$, and for $\tau_{L} / \tau_{D}=1, D_{S}^{\infty} / D_{T}^{\infty} \approx 8$. For uniformly distributed particle releases, the $D_{S}(t)$ ramp-up time increased with $\tau_{L} / \tau_{D}$. These results suggest that application of Taylor's asymptotic diffusivity in regions where the diffusive process has non-zero $\tau_{L}$ may result in a significant underestimate of the large-time shear-induced diffusivity. At a fixed $\tau_{L} / \tau_{D}$, the $D_{S}\left(t \mid y_{0}\right)$ ramp-up time was affected by $y_{0} / L$ (analogous to previous studies with $\tau_{L}=0$ ), and was largest when $y_{0} / L$ is in regions of low shear.

Many of the $D_{S}$ properties were explained with asymptotic analysis. For small $\tau_{L} / \tau_{D}$, the large-time shear-induced diffusivity has a small linear $\tau_{L} / \tau_{D}$ correction to the Taylor diffusivity and the $D_{S}(t)$ ramp-up time scale is $\tau_{D}$. For larger $\tau_{L} / \tau_{D}$, the ratio of the large-time shear-induced diffusivity to the Taylor diffusivity $D_{S}^{\infty} / D_{T}^{\infty}$ is a function of $\left(\tau_{L} / \tau_{D}\right)^{1 / 2}$ at leading order and the ramp-up time scale is $\tau_{S}=\left(\tau_{L} \tau_{D}\right)^{1 / 2}$. For $\tau_{L} / \tau_{D}>0.1$, the normalized curves $D_{S}(t) / D_{S}^{\infty}$ collapse onto the same function of $t / \tau_{S}$. The form of the large-time shear-induced diffusivity can be written as a squared velocity times a time scale $\tau$. By interpreting $\tau$ as the Lagrangian particle domaincrossing time, the leading-order asymptotic results are recovered. Moreover, the leading-order expression for the large-time shear-induced diffusivity $D_{S}^{\infty}$ is consistent with the turbulent shear dispersion diffusivity $\left(D \propto u_{*} L\right)$ when properties of wallbounded turbulence are considered.

These results demonstrate that shear-induced dispersion, for non-zero Lagrangian time-scale random velocities, has a significantly different large-time limit and time dependence than that for the well-studied classical zero-Lagrangian time-scale case.

\section{Acknowledgements}

We are grateful for discussions with B. Young and G. Ierley. Their expertise helped us in this work. In addition, we are also grateful to the anonymous reviewers who helped improve this work. We also thank to the National Science Foundation and the Office of Naval Research for funding this work.

\section{Appendix A. The subensemble-averaged squared velocity}

Here the derivation following Uhlenbeck \& Ornstein (1930) of the subensembleaveraged squared velocity (3.3) is presented. Other transverse statistics, in particular the squared displacements (3.7), (3.9) and (3.10), are similarly derived. First, the 
particle velocity along a trajectory is determined by integrating $(2.1 b)$ from 0 to $t_{1}$ to obtain

$$
v\left(t_{1} \mid v_{0}\right)=v_{0} \mathrm{e}^{-t_{1} / \tau_{L}}+\sqrt{\frac{2 \overline{v^{2}}}{\tau_{L}}} \mathrm{e}^{-t_{1} / \tau_{L}} \int_{0}^{t_{1}} \mathrm{e}^{t^{\prime} / \tau_{L}} w\left(t^{\prime}\right) \mathrm{d} t^{\prime}
$$

which after subensemble averaging gives (3.1) since $\left\langle w\left(t^{\prime}\right)\right\rangle=0$. The subensembleaveraged squared velocity is found from $\left\langle v\left(t_{1}\right) v\left(t_{2}\right)\right\rangle$, namely

$$
\begin{aligned}
\left\langle v\left(t_{2} \mid v_{0}\right) v\left(t_{1} \mid v_{0}\right)\right\rangle= & \left\langle\left[v_{0} \mathrm{e}^{-t_{2} / \tau_{L}}+\sqrt{\frac{2 \overline{v^{2}}}{\tau_{L}}} \mathrm{e}^{-t_{2} / \tau_{L}} \int_{0}^{t_{2}} \mathrm{e}^{t^{\prime} / \tau_{L}} w\left(t^{\prime}\right) \mathrm{d} t^{\prime}\right]\right. \\
& \left.\times\left[v_{0} \mathrm{e}^{-t_{1} / \tau_{L}}+\sqrt{\frac{2 \overline{v^{2}}}{\tau_{L}}} \mathrm{e}^{-t_{1} / \tau_{L}} \int_{0}^{t_{1}} \mathrm{e}^{t^{\prime \prime} / \tau_{L}} w\left(t^{\prime \prime}\right) \mathrm{d} t^{\prime \prime}\right]\right\rangle .
\end{aligned}
$$

Since the average of the random forcing is $\langle w\rangle=0$, and the noise covariance is $\left\langle w\left(t^{\prime}\right) w\left(t^{\prime \prime}\right)\right\rangle=\delta\left(t^{\prime}-t^{\prime \prime}\right)$, only two terms remain

$$
\begin{aligned}
\left\langle v\left(t_{2} \mid v_{0}\right) v\left(t_{1} \mid v_{0}\right)\right\rangle= & v_{0}^{2} \mathrm{e}^{-\left(t_{1}+t_{2}\right) / \tau_{L}}+\frac{2 \overline{v^{2}}}{\tau_{L}} \mathrm{e}^{-\left(t_{1}+t_{2}\right) / \tau_{L}} \\
& \times \int_{0}^{t_{1}} \int_{0}^{t_{1}} \mathrm{e}^{\left(t^{\prime}+t^{\prime \prime}\right) / \tau_{L}} \delta\left(t^{\prime}-t^{\prime \prime}\right) \mathrm{d} t^{\prime} \mathrm{d} t^{\prime \prime},
\end{aligned}
$$

where the $t^{\prime}$ limit is only to $t_{1}$ as $\delta\left(t^{\prime}-t^{\prime \prime}\right)$ is zero for all $t^{\prime}>t_{1}$ due to the assumption $t_{2}>t_{1}$. Changing variables to $a=t^{\prime}-t^{\prime \prime}$ and $b=t^{\prime}+t^{\prime \prime}$

$$
\left\langle v\left(t_{2} \mid v_{0}\right) v\left(t_{1} \mid v_{0}\right)\right\rangle=v_{0}^{2} \mathrm{e}^{-\left(t_{1}+t_{2}\right) / \tau_{L}}+\frac{\overline{v^{2}}}{\tau_{L}} \mathrm{e}^{-\left(t_{1}+t_{2}\right) / \tau_{L}} \int_{0}^{2 t_{1}} \int_{-\infty}^{\infty} \mathrm{e}^{b / \tau_{L}} \delta(a) \mathrm{d} a \mathrm{~d} b,
$$

where the $a$ integral is from $-\infty$ to $\infty$ due to the localized nature of $\delta(a)$. Performing the integrals gives

$$
\left\langle v\left(t_{2} \mid v_{0}\right) v\left(t_{1} \mid v_{0}\right)\right\rangle=v_{0}^{2} \mathrm{e}^{-\left(t_{1}+t_{2}\right) / \tau_{L}}+\overline{v^{2}}\left[\mathrm{e}^{\left(t_{1}-t_{2}\right) / \tau_{L}}-\mathrm{e}^{-\left(t_{1}+t_{2}\right) / \tau_{L}}\right]
$$

which when rearranged becomes (3.3). The position statistics are found similarly using the displacement

$$
y_{1}\left(t_{1} \mid v_{0}\right)=\int_{0}^{t_{1}}\left[v_{0} \mathrm{e}^{-t^{\prime} / \tau_{L}}+\sqrt{\frac{2 \overline{v^{2}}}{\tau_{L}}} \mathrm{e}^{-t^{\prime} / \tau_{L}} \int_{0}^{t^{\prime}} \mathrm{e}^{t^{\prime \prime} / \tau_{L}} w\left(t^{\prime \prime}\right) \mathrm{d} t^{\prime \prime}\right] \mathrm{d} t^{\prime} .
$$

Working from this expression and others like it, the position statistics $\left\langle y_{1}^{2}\left(t_{1} \mid v_{0}\right)\right\rangle$, $\left\langle y_{1}\left(t_{1} \mid v_{0}\right) y_{2}\left(t_{2} \mid v_{0}\right)\right\rangle$ and $\left\langle y_{2}^{2}\left(t_{2} \mid v_{0}\right)\right\rangle$ are found using the same techniques from which $\left\langle v\left(t_{1} \mid v_{0}\right) v\left(t_{2} \mid v_{0}\right)\right\rangle$ was derived above.

\section{Appendix B. Asymptotic analysis}

We find the asymptotic approximation to the integral in the shear-induced diffusivity $\int_{0}^{t} \exp \left(-\varsigma_{1}^{2} \gamma_{n}^{2} / 2\right) \mathrm{d} t_{1}$ using Taylor's asymptotic method of evaluating integrals. We start 
with the Taylor series expansion of $\varsigma^{2}$ in powers of $t$ :

$$
\frac{1}{2} \varsigma^{2} \gamma_{n}^{2} \approx \frac{n^{2} \pi^{2}}{2}\left(\frac{t}{\tau_{S}}\right)^{2}\left[1-\frac{1}{3}\left(\frac{t}{\tau_{L}}\right)+O\left(\frac{t}{\tau_{L}}\right)^{2}\right]
$$

where the shear time scale is $\tau_{S}=\left(\tau_{D} \tau_{L}\right)^{1 / 2}$. Clearly, this expansion has difficulties for $\tau_{L} \rightarrow 0$ since $\tau_{L}$ appears in the denominator of the expansion's leading term. Furthermore, (B 1) does not possess the proper $\tau_{L}=0$ limit of $\varsigma^{2} \gamma_{n}^{2} / 2=t / \tau_{D}$. Thus, the expansion (B 1) is only good for finite $\tau_{L}$, however, it is convergent for all non-zero $\tau_{L}$. The integral sought is then

$$
\int_{0}^{t} \mathrm{e}^{-\frac{1}{2} \sigma_{1}^{2} \gamma_{n}^{2}} \mathrm{~d} t_{1} \sim \tau_{S} \int_{0}^{t / \tau_{S}} \mathrm{e}^{-\frac{1}{2} n^{2} \pi^{2} t^{\prime 2}} \mathrm{e}^{\frac{1}{6} \pi^{2} n^{2} \alpha t^{\prime 3}-\frac{1}{24} \pi^{2} n^{2} \alpha^{2} t^{\prime 4}+O\left(\alpha^{3} t^{5}\right)} \mathrm{d} t^{\prime}
$$

where $t^{\prime}=t / \tau_{S}$ is non-dimensional and $\alpha=\left(\tau_{D} / \tau_{L}\right)^{1 / 2}$. Expanding the second exponential in a Taylor series yields

$$
\int_{0}^{t} \mathrm{e}^{-\frac{1}{2} \sigma_{1}^{2} \gamma_{n}^{2}} \mathrm{~d} t_{1} \sim \tau_{S} \int_{0}^{t / \tau_{S}} \mathrm{e}^{-\frac{1}{2} n^{2} \pi^{2} t^{\prime 2}}\left[1+\frac{1}{6} \pi^{2} n^{2} \alpha t^{\prime 3}-\frac{1}{24} \pi^{2} n^{2} \alpha^{2} t^{4}+O\left(\alpha^{3} t^{5}\right)\right] \mathrm{d} t^{\prime},
$$

and when integrated results in the asymptotic series for the integral

$$
\begin{aligned}
\int_{0}^{t} \mathrm{e}^{-\frac{1}{2} \sigma_{1}^{2} \gamma_{n}^{2}} \mathrm{~d} t_{1} \sim & \frac{\tau_{S}}{\sqrt{2 \pi}} \operatorname{erf}\left(\frac{n \pi t}{\sqrt{2} \tau_{S}}\right)\left[\frac{1}{n}-\frac{1}{6 n^{3} \pi^{2}} \alpha^{2}\right]+\frac{1}{3 n^{2} \pi^{2}} \alpha \\
& -\tau_{S} \mathrm{e}^{-\frac{1}{2} \pi^{2} n^{2}\left(\frac{t}{\tau_{S}}\right)^{2}}\left\{\left[\frac{1}{3 n^{2} \pi^{2}}+\frac{1}{6}\left(\frac{t}{\tau_{S}}\right)^{2}\right] \alpha\right. \\
& \left.+\left[\frac{1}{12 n^{2} \pi^{2}}\left(\frac{t}{\tau_{S}}\right)+\frac{1}{36}\left(\frac{t}{\tau_{S}}\right)^{3}+\frac{\pi^{2} n^{2}}{72}\left(\frac{t}{\tau_{S}}\right)^{5}\right] \alpha^{2}\right\}+O\left(\alpha^{3}\right) .
\end{aligned}
$$

up to order $O\left(\alpha^{2}\right)$. Keeping terms up to $O\left(\alpha^{3}\right)$ in the series, and letting $t \rightarrow \infty$, yields the asymptotic series for the large-time limit of the integral

$$
\int_{0}^{\infty} \mathrm{e}^{-\frac{1}{2} \sigma_{1}^{2} \gamma_{n}^{2}} \mathrm{~d} t_{1} \sim \tau_{S}\left(\frac{2^{1 / 2}}{2 n \pi^{1 / 2}}+\frac{1}{3 n^{2} \pi^{2}} \alpha+\frac{2^{1 / 2}}{24 n^{3} \pi^{5 / 2}} \alpha^{2}+\frac{4}{135 n^{4} \pi^{4}} \alpha^{3}+O\left(\alpha^{4}\right)\right)
$$

This is the crucial expression needed for evaluating the large-time shear-induced diffusivity $D_{S}^{\infty}$ in (5.5).

\section{REFERENCES}

ARIS, R. 1956 On the dispersion of a solute in a fluid flowing through a tube. Proc. R. Soc. Lond. A 235, 67-77.

BARTON, N. 1983 On the method of moments for solute dispersion. J. Fluid Mech. 126, 205-218.

Berloff, P. S. \& MCWilliams, J. C. 2002 Material transport in oceanic gyres. Part II: hierarchy of stochastic models. J. Phys. Ocean. 32 (3), 797-830.

CAMAssa, R., Lin, Z. \& MCLAughlin, R. M. 2010 The exact evolution of the scalar variance in pipe and channel flow. Commun. Math. Sci. 8, 601-626.

Castiglione, P. \& CRis Anti, A. 1999 Dispersion of passive tracers in a velocity field with non-delta-correlated noise. Phys. Rev. E 59, 3926-3934.

DAVIS, R. E. 1985 Drifter observation of coastal currents during CODE: the statistical and dynamical perspective. J. Geophys. Res. 90, 4756-4772. 
Dever, E. P., Hendershott, M. C. \& Winant, C. D. 1998 Statistical aspects of surface drifter observations of circulation in the Santa Barbara Channel. J. Geophys. Res. 103, 24,781-24,797.

Dewey, R. J. \& Sullivan, P. J. 1982 Longitudinal-dispersion calculations in laminar flows by statistical analysis of molecular motions. J. Fluid Mech. 125, 203-218.

Elder, J. W. 1959 The dispersion of marked fluid in turbulent shear flow. J. Fluid Mech. 5, $544-560$.

HABER, S. \& MAURI, R. 1988 Lagrangian approach to time-dependent laminar dispersion in rectangular conduits. Part 1. Two-dimensional flows. J. Fluid Mech. 190, 201-215.

Katayama, Y. \& Terauti, R. 1996 Brownian motion of a single particle under shear flow. Eur. J. Phys. 17, 136-140.

Latini, M. \& Bernoff, A. J. 2001 Transient anomalous diffusion in Poiseuille flow. J. Fluid Mech. 441, 399-411.

Li, T., Kheifets, S., Medellin, D. \& Raizen, M. G. 2010 Measurement of the instantaneous velocity of a Brownian particle. Science 328 (5986), 1673-1675.

McClean, J. L., Poulain, P. M. \& Pelton, J. W. 2002 Eulerian and Lagrangian statistics from surface drifters and a high-resolution POP simulation in the North Atlantic. J. Phys. Oceanogr. 32, 2472-2491.

Pavliotis, G. A. \& Stuart, A. M. 2005 Periodic homogenization for inertial particles. Physica D 204, 161-187.

Pavliotis, G., Stuart, A. M. \& Zygalakis, K. 2007 Homogenization for inertial particles in a random flow. Commun. Math. Sci. 5, 507-531.

Pavliotis, G. A., Stuart, A. M. \& Zygalakis, K. C. 2009 Calculating effective diffusivities in the limit of vanishing molecular diffusion. J. Comput. Phys. 228, 1030-1055.

Sallée, J. B., Speer, K., Morrow, R. \& Lumpkin, R. 2008 An estimate of Lagrangian eddy statistics and diffusion in the mixed layer of the Southern Ocean. J. Mar. Res. 66 (4), 441-463.

SAWfORD, B. L. \& YEUnG, P. K. 2001 Lagrangian statistics in uniform shear flow: direct numerical simulation and Lagrangian stochastic models. Phys. Fluids 13 (9), 2627-2634.

Spydell, M., Feddersen, F. \& GuZA, R. T. 2009 Observations of drifter dispersion in the surfzone: the effect of sheared alongshore currents. J. Geophys. Res. 114.

TAYlor, G. I. 1922 Diffusion by continuous movements. Proc. Lond. Math. Soc. 20, 196-212.

TAYLOR, G. I. 1953 Dispersion of soluble matter in solvent flowing slowly through a tube. Proc. Roy. Soc. A 219, 186-203.

TAYLOR, G. I. 1954 The dispersion of matter in turbulent flow through a pipe. Proc. Roy. Soc. A 223, 446-468.

Tennekes, H. \& Lumley, J. L. 1972 A First Course in Turbulence. MIT Press.

Uhlenbeck, G. E. \& Ornstein, L. S. 1930 On the theory of the Brownian motion. Phys. Rev. 36, 823-841.

VAn Den BRoeck, C. 1982 A stochastic description of longitudinal dispersion in uniaxial flows. Physica A 112A, 343-352.

Wilson, J. D. \& SAWFORD, B. L. 1996 Review of Lagrangian stochastic models for trajectories in the turbulent atmosphere. Boundary-Layer Meteorol. 78 (1), 191-210.

Young, W. R. \& Jones, S. 1991 Shear dispersion. Phys. Fluids A 3 (5), 1087-1101.

ZAMbianchi, E. \& GRIFFA, A. 1994 Effects of finite scales of turbulence on dispersion estimates. J. Mar. Res. 52, 129-148.

Zhurbas, V. 2003 Lateral diffusivity and Lagrangian scales in the Pacific Ocean as derived from drifter data. J. Geophys. Res. 108 (C5). 